\title{
THE INHOMOGENEOUS MINIMA OF BINARY QUADRATIC FORMS (II).
}

\author{
By \\ E. S. BARNES and H. P. F. SWINNERTON-DYER \\ of Trinity College, CAMbridge.
}

\section{Introduction.}

This paper continues the work of our previous paper ${ }^{1}$, with which we shall assume that the reader is familiar. There we were mainly concerned with finding the inhomogeneous minimum $M(f)$ of a rational indefinite binary quadratic form; and occasionally the methods yielded also the second minimum $M_{2}(f)$. We show here that our methods may be extended to deal with the problem of finding an enumerably infinite sequence of minima.

This problem has been solved for the particular forms $x^{2}+x y-y^{2}$ and $x^{2}-2 y^{2}$, by Davenport [1] and Varnavides [2] respectively. The method used by these authors is synthetic, and has the disadvantage of giving no information on the values of $M(f, P)$ close to (but less than) the limiting value $M^{\prime}==\overline{\lim } M(f, P)$.

We consider in sections 1 and 2 the norm-forms $x^{2}-11 y^{2}$ and $x^{2}+x y-3 y^{2}$. The first of these was chosen as the simplest form whose second minimum was not easily established by the methods of our previous paper. We obtain for it, in Theorem 1, a result precisely analogous to those found by Davenport and Varnavides, with an additional clause on the existence of a non-enumerable infinity of incongruent points $P$ with $M(f, P)>M^{\prime}-\varepsilon$; and we may regard this as an entirely typical result.

In Theorem 7 of our previous paper, we proved that for the form $x^{2}+x y-3 y^{2}$ the first minimum $M(f)$ is taken at both rational and irrational points; its behaviour might therefore be expected not to conform to the usual pattern. We show in fact,

1 "The inhomogeneous minima of binary quadratic forms (I)", Acta Math. Vol. 87, 1952. References to literal theorems and to the bibliography refer to this paper. 
in Theorem 2, that the enumerable sequence of minima extends below $M^{\prime}$, and establish the complete enumerable sequence.

The proof of these two theorems uses the ideas and general results of our previous paper. It should be noted, however, that the fundamental concept is not that of a point but of a point-series, i.e. a point and all its transforms under positive and negative powers of the fundamental automorph $T$. This distinction was not made previously, since we were mainly concerned with points fixed under a small power of $T$.

In section 3 we establish two general results on the set of values of $M(f, P)$, and make some conjectures. Finally, in section 4, we indicate how our methods may be extended to higher dimensions.

We wish to express our indebtedness to Dr. J. W. S. Cassels for his criticisms and encouragement.

1. Throughout this section we shall write

$$
\begin{gathered}
f(x, y)=x^{2}-11 y^{2}, \quad \xi=x+y \sqrt{11}, \quad \eta=x-y \sqrt{11} . \\
\tau=10+3 \sqrt{11}=19.9498 \ldots, \quad \tau^{\prime}=10-3 \sqrt{11}=0.0501 \ldots, \\
T=\left(\begin{array}{rr}
10 & 33 \\
3 & 10
\end{array}\right),
\end{gathered}
$$

so that $T$ is the fundamental automorph of $f(x, y)$ and $\tau$ the fundamental unit of the field $k(\sqrt{11})$, and we have $f=\xi \eta, \tau \tau^{\prime}=1$.

Theorem 1. With the notation above, we have

$$
\begin{gathered}
M_{1}(f)=\frac{19}{22}, \\
M_{2}(f)=\frac{3125}{3971}, \\
M_{k}(f)=\frac{19}{22}-\frac{325}{209} \mu_{k-3}-\frac{1}{7942} \mu_{k-3}^{2}, \quad(k=3,4, \ldots)
\end{gathered}
$$

where

$$
\begin{gathered}
\mu_{k}=\frac{\tau^{3 k+3}-\tau^{-3 k-3}}{\tau^{3 k+4}-\tau^{-3 k-4}}, \\
M_{\infty}(f)=\lim _{k \rightarrow \infty} M_{k}(f)=\frac{5(3711 \sqrt{11}-11684)}{3971}=0.785689 \ldots
\end{gathered}
$$


All these minima are attained, and the points at which $M(f, P)=M_{k}(f)$ form a pair of conjugate point series of finite order, generated by the points $\pm P_{k}$ where

$$
\begin{gathered}
P_{1}=\left(\frac{1}{2}, \frac{7}{22}\right), \\
P_{2}=\left(0, \frac{98}{209}\right) \\
P_{k}=\left(\frac{1-\mu_{k-3}}{2}, \frac{133-63 \mu_{k-3}}{418}\right) \quad(k=3,4, \ldots),
\end{gathered}
$$

(except that for $k=1,2$ the two series coincide). There are thus 2 distinct points (mod 1 ) for which $M(f, P)=M_{1}, 6$ for which $M(f, P)=M_{2}$ and $4(3 k-5)$ for which $M(f, P)=$ $=M_{k}(k=3,4, \ldots)$. The points for which $M(f, P)=M_{\infty}$ form two conjugate infinite point series generated by the points $\pm P_{\infty}$ where

$$
P_{\infty}=\left(\frac{3 \sqrt{11}-9}{2}, \frac{189 \sqrt{11}-497}{418}\right) .
$$

However, for any fixed $\varepsilon>0$, the set of points $P$ for which $M_{\infty}(f)>M(f, P)>$ $>M_{\infty}(f)-\varepsilon$ has the cardinal number of the continuum, and includes an infinity of rational points.

These results are exactly analogous to those for $x^{2}+x y-y^{2}$ and $x^{2}-2 y^{2}$, though Davenport and Varnavides have not given the results corresponding to the last two sentences of Theorem 1. In order to make the argument clearer, each main step has been stated as a separate lemma. The lemmas represent, however, successive steps in a single argument, rather than distinct results which have to be combined to prove the theorem.

We shall assume the portion of Theorem 1 contained in ( 1 ) and (6), which is in fact the special case $n=1$ of Theorem 4 of our previous paper. We could clearly obtain this again with the rest of Theorem 1, but it is convenient to have the precise upper bound for $M(f, P)$, in the application of Theorem $\mathrm{B}$.

Lemma 1. If $M(f, P)>0.78$, then $P$ lies $(\bmod 1)$ in one of the three regions

$$
\begin{aligned}
& \boldsymbol{R}_{1}^{\prime}: 1.4554<\xi<1.7125, \quad-0.7125<\eta<-0.4554, \\
& \boldsymbol{R}_{2}^{\prime}: 2.6041<\xi<2.8613, \quad-1.8613<\eta<-1.6041, \\
& \boldsymbol{R}_{3}^{\prime}: 1.5221<\xi<1.7945, \quad-1.7945<\eta<-1.5221 .
\end{aligned}
$$

Moreover, the same is true for $T^{n} P$ for every integer $n$. 
The number 0.78 has no special significance; we could equally choose any number near to but less than $M_{\infty}(f)$. This will be clearer after Lemma 3 , in which the bounds for the three regions are refined to values which appear natural in the problem and which do not directly depend (as the bounds above do) on the constant 0.78 .

The regions $R_{1}^{\prime}, R_{2}^{\prime}$ are each symmetric about $x=\frac{1}{2}$ and are images in $y=\frac{1}{2}$; the region $\mathcal{R}_{3}^{\prime}$ is symmetric about $x=0$ and about $y=\frac{1}{2}$. It is therefore sufficient to prove the lemma for points of the region $0 \leq x, y \leq \frac{1}{2}$, since it can then be extended to the whole unit square by suitable reflections.

Suppose that

$$
P=(x, y) \text { has } M(f, P)>0.78 \text { and } 0 \leq x, y \leq \frac{1}{2} .
$$

It is impossible that $f(x-1, y)>0.78$ for this would require

and therefore

$$
0 \leq 11 y^{2}=(x-1)^{2}-f(x, y)<1-0.78=0.22
$$

$$
|f(x, y)|=\left|x^{2}-11 y^{2}\right| \leq \max \left(\frac{1}{4}, 0.22\right)<0.78 \text {. }
$$

Thus we may take $f(x-1, y)<-0.78$, which is

$$
(\xi-1)(\eta-1)<-0.78
$$

We now consider the two possible alternatives for $f(x+1, y)$ :

$$
\text { (i) } f(x+1, y)=(x+1)^{2}-11 y^{2}>0.78 \text {. }
$$

Then $11 y^{2}<(x+1)^{2}-0.78 \leq 2.25-0.78=1.47$, so that $\xi<\frac{1}{2}+\sqrt{1.47}<1.7125$. Now since $(\xi+1)(\eta+1)=f(x+1, y)>0.78$, this gives

$$
\eta>\frac{0.78}{2.7125}-1>0.2875-1=-0.7125 \text {. }
$$

Now it follows from (10) that $\xi>\frac{0.78}{1-\eta}+1>\frac{0.78}{1.7125}+1>1.4554$, and since $x \leq \frac{1}{2}$, $11 y^{2} \geq(1-x)^{2}+0.78 \geq 1.03$, and so

$$
\eta=x-y \sqrt{11} \leq 0.5-\sqrt{1.03}<-0.5148 .
$$

These bounds on $\xi, \eta$ together show that $(x, y)$ lies in $R_{1}^{\prime}$.

(ii) $f(x+1, y)=(\xi+1)(\eta+1)<-0.78$.

We have trivially $\xi \leq \xi\left(\frac{1}{2}, \frac{1}{2}\right)<2.1583$, and therefore

$$
\eta+1<\frac{-0.78}{3.1583}, \quad \eta<-1.2469 .
$$


Now $f(x-2, y-1)=(2+\sqrt{11}-\xi)(2-\sqrt{11}-\eta)$. If this is negative, the second factor must be so and we have

$$
|f(x-2, y-1)|<(2+\sqrt{11})(\sqrt{11}-2-1.2469)<5.3167 \times 0.0698<0.78 .
$$

But this is impossible, and we therefore have

$$
f(x-2, y-1)>0.78
$$

This gives $11(1-y)^{2}<(2-x)^{2}-0.78 \leq 3.22$, whence $y \sqrt{11}>1.5221$,

$$
\xi=x+y \sqrt{11}>1.5221 \text {. }
$$

Substituting this back in (11) we find

$$
2-\sqrt{11}-\eta>\frac{0.78}{2+\sqrt{11}-\xi}>\frac{0.78}{3.7946}>0.2055, \quad \eta<-1.5221
$$

On the other hand, it is trivial that $\eta \geq-\frac{1}{2} \sqrt{11}>-1.6584$; and since $\xi-\eta=$ $=2 y \sqrt{11} \leq \sqrt{11}$, we have $\xi<1.7945$. These bounds on $\xi$. $\eta$ together show that $(x, y)$ lies in $\boldsymbol{R}_{3}^{\prime}$.

This proves the Lemma.

These bounds are very rough, however, and we have next to improve them as much as possible. For this we must know something about the behaviour of $T^{n} P$ for given $P$.

Lemma 2. Let $M(f, P)>0.78$. Then, using $(x, y)$ co-ordinates,

(i) if $P \in R_{1}^{\prime}, \quad T P \in R_{2}^{\prime}+(15,4)$ or $T P \in R_{3}^{\prime}+(15,4)$;

(ii) if $P \in \mathcal{R}_{2}^{\prime}, \quad T P \in R_{1}^{\prime}+(27,8)$ or $T P \in R_{3}^{\prime}+(28,8)$;

(iii) if $P \in R_{3}^{\prime}, \quad T P \in R_{1}^{\prime}+(17,5)$ or $T P \in R_{2}^{\prime}+(15,4)$.

There are similar results for $\dot{T}^{-1} P$, which may be deduced from these immediately.

The bounds in Lemma 1 give us:

$$
\left.\begin{array}{lll}
\text { In } R_{1}^{\prime}, & 0.3714<x<0.6286, & 0.2879<y<0.3655 ; \\
\text { In } R_{2}^{\prime}, \quad 0.3714<x<0.6286, & 0.6345<y<0.7121 ; \\
\text { In } R_{3}^{\prime}, \quad-0.1362<x<0.1362, & 0.4589<y<0.5411 .
\end{array}\right\}
$$

The transformation $T$ is equivalent to multiplying $\xi$ and dividing $\eta$ by $\tau$. Thus $T \boldsymbol{R}_{1}^{\prime}$ is $29.0349<\xi<34.1643,-0.0358<\eta<-0.0228$ whence, since $\xi-\eta=2 y \sqrt{11}$, 
4.3806<y<5.1559. Now from Lemma $1, T P \in \boldsymbol{R}_{i}^{\prime}+\left(x_{0}, y_{0}\right)$ for $i=1,2$ or 3 and some integers $x_{0}, y_{0}$. Comparing the bound here for $y$ with (12) we see that

$$
\text { either } \quad i=2, y_{0}=4 \quad \text { or } \quad i=3, y_{0}=4 \text {. }
$$

Moreover, since $T P-\left(x_{0}, y_{0}\right) \in \mathcal{R}_{i}^{\prime}, \eta(T P)+y_{0} \sqrt{11}-x_{0}$ must be an admissible value for $\eta$ in $\mathcal{R}_{i}$, and a comparison with Lemma 1 gives (i) of Lemma 2.

(ii) now follows from (i) by simple reflexion in the point $\left(\frac{1}{2}, \frac{1}{2}\right)$.

$T \boldsymbol{R}_{3}^{\prime}$ is $30.3655<\xi<35.8001,-0.0900<\eta<-0.0762$, from which it follows that $4.5892<y<5.4107$. Comparing with (12) and then considering possible values of $\eta$ as above, we now obtain (iii) of Lemma 2.

Lemma 3. If $M(f, P)>0.78$, then $P$ lies $(\bmod 1)$ in one of the three regions

$$
\begin{aligned}
& R_{1}: \lambda_{2} \leq \xi \leq \lambda_{1}, \quad 1-\lambda_{1} \leq \eta \leq 1-\lambda_{2} ; \\
& R_{2}: 1+\sqrt{11}-\lambda_{1} \leq \xi \leq 1+\sqrt{11}-\lambda_{2}, \quad \lambda_{2}-\sqrt{11} \leq \eta \leq \lambda_{1}-\sqrt{11} ; \\
& R_{3}: \sqrt{11}-\lambda_{3} \leq \xi \leq \lambda_{3}, \quad-\lambda_{3} \leq \eta \leq \lambda_{3}-\sqrt{11} ;
\end{aligned}
$$

where

$\lambda_{1}=\frac{67+39 \sqrt{11}}{126}=1.55832 \ldots, \lambda_{2}=\frac{59+39 \sqrt{11}}{126}=1.49482 \ldots, \lambda_{3}=\frac{13+63 \sqrt{11}}{126}=1.76148 \ldots$

Each region $\boldsymbol{R}_{i}$ is contained in the corresponding $\boldsymbol{R}_{i}^{\prime}$ of Lemma 1.

Corollary. Lemma 2 still holds if every $\boldsymbol{R}_{i}^{\prime}$ is replaced by the corresponding $\boldsymbol{R}_{i}$.

The point series defined by (1) and (6) has no points in $\mathfrak{R}_{3}^{\prime}$, and satisfies the conditions of Lemma 2. Thus the two cases

$$
P \in \mathfrak{R}_{1}^{\prime}, \quad T P \in \boldsymbol{R}_{2}^{\prime}+(15,4) \text { and } P \in \mathcal{R}_{2}^{\prime}, \quad T P \in \boldsymbol{R}_{1}^{\prime}+(27,8)
$$

of Lemma 2 are certainly possible, and there exist points in $\boldsymbol{R}_{1}^{\prime}, \mathfrak{R}_{2}^{\prime}$ with $M(f, P)>0.78$. First, if there are no points in $\mathcal{R}_{3}^{\prime}$ with $M(f, P)>0.78$, then only the two cases (13) of Lemma 2 can occur, and we can easily deduce from them that if $P \in R_{1}^{\prime}, T^{-1} P \in$ $\in \mathcal{R}_{2}^{\prime}+(-6,1)$, and that if $P \in \boldsymbol{R}_{2}^{\prime}, T^{-1} P \in \boldsymbol{R}_{1}^{\prime}+(-18,5)$. Now Theorem $\mathrm{D}^{\prime}$ shows that the only points with $M(f, P)>0.78$ are those given by $(6)$, in which case Lemma 3 can easily be verified.

Hence we may assume ${ }^{1}$ that $\mathcal{R}_{3}^{\prime}$ contains points with $M(f, P)>0.78$. Let the

1 We could also have shown this from the point series generated by (7), but this would have been anticipating the subsequent course of the proof. 
upper and lower bounds of $\xi$, taken over all $P \in R_{1}^{\prime}$ with $M(f, P)>0.78$, be $\lambda_{1}^{\prime}$ and $\lambda_{2}^{\prime}$ respectively. By symmetry, the bounds for $\eta$ in $\boldsymbol{R}_{1}^{\prime}$, and for $\xi, \eta$ in $\boldsymbol{R}_{2}^{\prime}$, must take the forms given in Lemma 3 , writing $\lambda_{i}^{\prime}$ for $\lambda_{i}$ throughout. Similarly, if $\lambda_{3}^{\prime}$ is the upper bound of $\xi$ over all $P \in R_{3}^{\prime}$ with $M(f, P)>0.78$, the remaining bounds in $\widehat{R}_{3}^{\prime}$ must take the form shown. We have from Lemma 1

$$
1.7125>\lambda_{1}^{\prime} \geq \lambda_{2}^{\prime}>1.4554, \quad 1.7945>\lambda_{3}^{\prime} \geq \frac{1}{2} \sqrt{11}>1.6583 .
$$

Now it follows from Lemma 2 that

$$
\begin{aligned}
& \operatorname{Min}\left[16+5 \sqrt{11}-\lambda_{1}^{\prime}, 15+5 \sqrt{11}-\lambda_{3}^{\prime}\right] \leq \tau \lambda_{2}^{\prime}, \\
& \tau \lambda_{1}^{\prime} \leq \operatorname{Max}\left[16+5 \sqrt{11}-\lambda_{2}^{\prime}, 15+4 \sqrt{11}+\lambda_{3}^{\prime}\right] ; \\
& \operatorname{Min}\left[27+8 \sqrt{11}+\lambda_{2}^{\prime}, 28+9 \sqrt{11}-\lambda_{3}^{\prime}\right] \leq \tau\left(1+\sqrt{11}-\lambda_{1}^{\prime}\right), \\
& \tau\left(1+\sqrt{11}-\lambda_{2}^{\prime}\right) \leq \operatorname{Max}\left[27+8 \sqrt{11}+\lambda_{1}^{\prime}, 28+8 \sqrt{11}+\lambda_{3}^{\prime}\right] ; \\
& \operatorname{Min}\left[17+5 \sqrt{11}+\lambda_{2}^{\prime}, 16+5 \sqrt{11}-\lambda_{1}^{\prime}\right] \leq \tau\left(\sqrt{11}-\lambda_{3}^{\prime}\right), \\
& \tau \lambda_{3}^{\prime} \leq \operatorname{Max}\left[17+5 \sqrt{11}+\lambda_{1}^{\prime}, 16+5 \sqrt{11}-\lambda_{2}^{\prime}\right] .
\end{aligned}
$$

We may resolve the Max, Min by (14), and we find that these six inequalities become

$$
\left.\begin{array}{l}
\tau \lambda_{1}^{\prime}+\lambda_{2}^{\prime} \leq 16+5 \sqrt{11}, \\
\tau \lambda_{2}^{\prime}+\lambda_{3}^{\prime} \geq 15+5 \sqrt{11}, \\
\tau \lambda_{3}^{\prime}-\lambda_{1}^{\prime} \leq 17+5 \sqrt{11},
\end{array}\right\}
$$

each twice repeated. Eliminating any two of $\lambda_{1}^{\prime}, \lambda_{2}^{\prime}, \lambda_{3}^{\prime}$, we deduce that

$$
\lambda_{1}^{\prime} \leq \frac{67+39 \sqrt{11}}{126}, \quad \lambda_{2}^{\prime} \geq \frac{59+39 \sqrt{11}}{126}, \quad \lambda_{3}^{\prime} \leq \frac{13+63 \sqrt{11}}{126}
$$

and these results prove the first sentence of Lemma 3. The rest now follows by comparing these bounds with those of Lemma 1 .

The Corollary is trivial. Since we shall henceforth work with the regions $\mathcal{R}_{i}$ of Lemma 3 , rather than the $\mathcal{R}_{i}^{\prime}$ of Lemma 1 , future references to Lemma 2 will be to it as affected by the corollary.

We note that the $\lambda_{i}$ satisfy the equations (15) with equality, i.e.

$$
\left.\begin{array}{c}
\tau \lambda_{1}+\lambda_{2}=16+5 \sqrt{11}, \quad \tau \lambda_{2}+\lambda_{3}=15+5 \sqrt{11}, \\
\tau \lambda_{3}-\lambda_{1}=17+5 \sqrt{11} .
\end{array}\right\}
$$

It is convenient to break up $\boldsymbol{R}_{3}$ into smaller regions. For this purpose we define $\boldsymbol{R}_{i j}(i, j=1,2)$ as the set of points $P$ for which $T^{-1} P \in \boldsymbol{R}_{t}(\bmod 1), P \in \boldsymbol{R}_{3}, T P \in \boldsymbol{R}_{j}$ 
(mod 1). It follows from Lemmas 2 and 3 that if $M(f, P)>0.78$ and $P \in R_{3}$, then $P$ lies in one of the four $R_{i j}$. We next show the stronger

Lemma 4. If $M(f, P)>0.78, P \in \widehat{R}_{3}$ then $P \in \mathfrak{R}_{11}$ or $P \in \boldsymbol{R}_{32}$. These regions are

$$
\begin{array}{ll}
\boldsymbol{R}_{11}: \sqrt{11}-\lambda_{1} \leq \xi \leq \lambda_{3}, & -\lambda_{3} \leq \eta \leq \lambda_{1}-\sqrt{11} ; \\
\mathcal{R}_{22}: \sqrt{11}-\lambda_{3} \leq \xi \leq \lambda_{1}, & -\lambda_{1} \leq \eta \leq \lambda_{3}-\sqrt{11} .
\end{array}
$$

By Lemma 2, $\boldsymbol{R}_{11}$ is the set of points common to $T \boldsymbol{R}_{1}-(15,4), \boldsymbol{R}_{3}$ and $T^{-1} \boldsymbol{R}_{1}+$ $+(5,-1)=T^{-1}\left[\boldsymbol{R}_{1}+(17,5)\right]$. Now from Lemma $3, T \boldsymbol{R}_{1}-(15,4)$ is

$$
\begin{aligned}
\tau \lambda_{2}-15-4 \sqrt{11} \leq \xi \leq \tau \lambda_{1}-15-4 \sqrt{11}, \\
\tau^{-1}\left(1-\lambda_{1}\right)-15+4 \sqrt{11} \leq \eta \leq \tau^{-1}\left(1-\lambda_{2}\right)-15+4 \sqrt{11},
\end{aligned}
$$

which reduce by (16) to

$$
\sqrt{11}-\lambda_{3} \leq \xi \leq 1+\sqrt{11}-\lambda_{2}, \quad-\lambda_{3} \leq \eta \leq \lambda_{1}-\sqrt{11} .
$$

Similarly $T^{-1} \boldsymbol{R}_{1}+(5,-1)$ is

$$
\tau^{-1} \lambda_{2}+5-\sqrt{11} \leq \xi \leq \tau^{-1} \lambda_{1}+5-\sqrt{11}, \quad \tau\left(1-\lambda_{1}\right)+5-\sqrt{11} \leq \eta \leq \tau\left(1-\lambda_{2}\right)+5+\sqrt{11},
$$

which reduce to

Also $R_{3}$ is

$$
\sqrt{11}-\lambda_{1} \leq \xi \leq \lambda_{3}, \quad \lambda_{2}-1-\sqrt{11} \leq \eta \leq \lambda_{3}-\sqrt{11} .
$$

$$
\sqrt{11}-\lambda_{3} \leq \xi \leq \lambda_{3}, \quad-\lambda_{3} \leq \eta \leq \lambda_{3}-\sqrt{11} .
$$

A comparison of these three results gives the bounds for $\boldsymbol{R}_{11}$ stated in the Lemma. We may in the same way obtain the bounds for $\boldsymbol{R}_{22}$ above and those for $\boldsymbol{R}_{12}, \boldsymbol{R}_{21}$ below:

$$
\begin{array}{ll}
R_{12}: \sqrt{11}-\lambda_{3} \leq \xi \leq \lambda_{1}, & -\lambda_{3} \leq \eta \leq \lambda_{1}-\sqrt{11} ; \\
\mathcal{R}_{21}: \sqrt{11}-\lambda_{1} \leq \xi \leq \lambda_{3}, & -\lambda_{1} \leq \eta \leq \lambda_{3}-\sqrt{11} .
\end{array}
$$

Now consider 1 in $R_{12}, f(x+5, y-2)=(\xi+5-2 \sqrt{11})(\eta+5+2 \sqrt{11})$. Since $\xi \leq \lambda_{1}<$ $<2 \sqrt{11}-5<1.6333$, we have

$$
\begin{aligned}
|f(x+5, y-2)| & <\left(\lambda_{3}+\sqrt{11}-5\right)\left(\lambda_{1}+5+\sqrt{11}\right) \\
& <0.07812 \times 9.875 \\
& =0.771435<0.78
\end{aligned}
$$

1 To consider this apparently obscure hyperbola is equivalent to considering $f(x-1, y-1)$ for the point in $\mathcal{R}_{2}$ congruent to $T P$. That this last is a natural thing to do will be clear from the proof of Lemma 8 below. 
Thus $M(f, P)<0.78$ for $P \in R_{12}$; by reflexion in $\left(0, \frac{1}{2}\right)$, the same result holds for $P \in R_{21}$; this completes the proof of the Lemma.

We now define a point $P$ as admissible if, for each $n, T^{n} P$ lies $(\bmod 1)$ in some $\boldsymbol{R}_{i}(i=1,2,11,22)$ and either $P \in \boldsymbol{R}_{1}$ or $P \in \boldsymbol{R}_{22}$. By Lemmas 3 and 4 , for any $P$ with $M(f, P)>0.78$ there is just one admissible $P^{*}= \pm P(\bmod 1)$. We now define, for all admissible $P$,

$$
\alpha_{n}=\alpha_{n}(P)= \begin{cases}1 \text { if } T^{n+1} P \in \boldsymbol{R}_{11} & \text { or } \boldsymbol{R}_{22}(\bmod 1), \\ 0 \text { if } T^{n+1} P \in \boldsymbol{R}_{1} & \text { or } \boldsymbol{R}_{2}(\bmod 1) .\end{cases}
$$

It is clear that to every admissible $P$ there corresponds the doubly infinite sequence $\left\{\alpha_{n}\right\}$ of elements 0 and 1 , in which two consecutive terms eannot both be 1 . Conversely we now show:

Lemma 5. Given any doubly-infinite sequence of elements 0,1 , such that no two consecutive elements are both 1 , there is an admissible $P$ which has this sequence for its $\left\{\alpha_{n}\right\}$, and $P$ is uniquely defined.

For the proof of this Lemma, we need the intermediate

Lemma 6. Consider any one of the six following cases:
(i) $P \in R_{1}, \quad T P \in R_{2}+(15,4)$;
(ii) $P \in R_{2}, \quad T P \in R_{1}+(27,8)$;
(iii) $P \in R_{1}, \quad T P \in R_{11}+(15,4)$;
(iv) $P \in R_{2}, \quad T P \in R_{22}+(28,8)$;
(v) $P \in R_{11}, \quad T P \in R_{1}+(17,5)$;
(vi) $P \in R_{22}, \quad T P \in R_{2}+(15,4)$.

In each case, the set of points $P$ satisfying these conditions forms a parallelogram with sides $\xi=$ const, $\eta=$ const; the bounds on $\xi$ are those given by the condition on $T P$, and the bounds on $\eta$ are those given by the condition on $P$.

(i) and (ii) follow at once from (16); (iii) and (v) from (17), (18) and the expression for $\boldsymbol{R}_{\mathbf{1 1}}$ in Lemma 4 ; (iv) and (vi) by symmetry from (iii) and (v) respectively.

Now we could clearly write in each case of Lemma $6 T P \in \mathcal{R}_{i}(\bmod 1)$ instead of $T P \in \boldsymbol{R}_{i}+\left(x_{i}, y_{i}\right)$, for we can obtain the $x_{i}, y_{i}$ from Lemma 2. Let us now write $P^{(n)}$ for the point congruent to $T^{n} P(\bmod 1)$ which lies in one of $\boldsymbol{R}_{1}, \boldsymbol{R}_{2}, \boldsymbol{R}_{11}, \boldsymbol{R}_{22}$. Lemma 6 now states that if the conditions $P \in \mathcal{R}_{i_{0}}, P^{(1)} \in \mathcal{R}_{i_{1}}$, are compatible (as given by Lemma 2 , or restated as the six cases of Lemma 6 ), then the only restrictions they put on $P$ are the bounds for $\xi$ obtained from $\boldsymbol{R}_{i_{1}}$ and those for $\eta$ obtained from $\mathcal{R}_{i_{0}}$. It follows from this by induction that if in the doubly infinite sequence of conditions $P^{(n)} \in \mathcal{R}_{i_{n}}(-\infty<n<\infty)$ every consecutive two are compatible, then all 
are compatible and define $P$ uniquely, the $\xi$ co-ordinate being given by the behaviour of $P^{(n)}$ as $n \rightarrow+\infty$ and the $\eta$ co-ordinate by the behaviour of $P^{(n)}$ as $n \rightarrow-\infty$.

To prove Lemma 5 , it remains to show that the sequence $\left\{\alpha_{n}\right\}$ of elements 0 and 1 corresponds to just one doubly infinite sequence of conditions on the $P^{(n)}$ compatible in pairs. Now, from Lemma 2 or Lemma 6 , we must have $i_{n}=1$ or 22 for $n$ even, $i_{n}=2$ or 11 for $n$ odd. It follows at once that the only possible sequence of $i_{n}$ is

$$
i_{n}=\left\{\begin{aligned}
& 1 \text { if } \alpha_{n-1}=0, n \text { even } \\
& 2 \text { if } \alpha_{n-1}=0, n \text { odd } \\
& 11 \text { if } \alpha_{n-1}=1, n \text { odd } \\
& 22 \text { if } \alpha_{n-1}=1, n \text { even }
\end{aligned}\right.
$$

and that with this definition, the conditions on two consecutive $P^{(n)}$ are compatible (remembering that two consecutive $i_{n}$ cannot both be 1 ). This proves the Lemma.

Lemmma 7. Let $P$ be admissible. Then using $(\xi, \eta)$ co-ordinates, $P$ is given by $\left(\frac{1}{2}+\frac{7}{22} \sqrt{11}+\sum_{1}^{\infty}(-\tau)^{-n} \alpha_{n-1}, \frac{1}{2}-\frac{7}{22} \sqrt{11}-\sum_{1}^{\infty}(-\tau)^{1-n} \alpha_{-n}\right)$. Further, $P^{(n)}$ is given by

$$
\left(\mu_{1}+(-1)^{n} \sum_{m=1}^{\infty}(-\tau)^{-m} \alpha_{m+n-1}, \mu_{2}-(-1)^{n} \sum_{m=1}^{\infty}(-\tau)^{1-m} \alpha_{n-m}\right)
$$

where $\mu_{1}, \mu_{2}$ are given by

$$
\begin{aligned}
& P^{(n)} \in R_{1} \text { or } R_{22}: \mu_{1}=\frac{1}{2}+\frac{7}{22} \sqrt{11}, \quad \mu_{2}=\frac{1}{2}-\frac{7}{22} \sqrt{11} ; \\
& P^{(n)} \in \mathcal{R}_{2}: \quad \mu_{1}=\frac{1}{2}+\frac{15}{22} \sqrt{11}, \quad \mu_{2}=\frac{1}{2}-\frac{15}{22} \sqrt{11} \text {; } \\
& P^{(n)} \in R_{11}: \quad \mu_{1}=-\frac{1}{2}+\frac{15}{22} \sqrt{11}, \mu_{2}=-\frac{1}{2}-\frac{15}{22} \sqrt{11} .
\end{aligned}
$$

Let the point given by the formula be $\bar{P}^{(n)}=\left(\bar{\xi}_{n}, \bar{\eta}_{n}\right)$. Then after the uniqueness clause of Lemma 5 it is enough to prove that

and

(i) $\bar{P}^{(n)}$ lies in the same $\mathcal{R}_{i_{n}}$ as $P^{(n)}$,

$$
\text { (ii) if } \bar{P}^{(n)}=P^{(n)} \text {, then } \bar{P}^{(n+1)}=P^{(n+1)} \text {, and conversely. }
$$

If $P^{(n)} \in R_{1}$, then $n$ is even and $\alpha_{n-1}=0$. Hence

$$
\frac{\tau^{-2}}{1-\tau^{-2}}=\sum_{1}^{\infty} \tau^{-2 m} \geq \bar{\xi}_{n}-\mu_{1} \geq \frac{-\tau^{-1}}{1-\tau^{-2}}=-\sum_{1}^{\infty} \tau^{1-2 m}
$$




$$
\frac{\tau^{-1}}{1-\tau^{-2}}=\sum_{1}^{\infty} \tau^{1-2 m} \geq \bar{\eta}_{n}-\mu_{2} \geq-\sum_{1}^{\infty} \tau^{-2 m}=\frac{-\tau^{-2}}{1-\tau^{-2}}
$$

These reduce to $\frac{31}{66} \sqrt{11} \geq \bar{\xi}_{n} \geq \frac{1}{2}+\frac{10}{33} \sqrt{11}, \frac{1}{2}-\frac{10}{33} \sqrt{11} \geq \bar{\eta}_{n} \geq 1-\frac{31}{66} \sqrt{11}$, so that by Lemma 3 , $\bar{P}^{(n)} \in R_{1}$. Similarly for the other three regions.

Now suppose $P^{(n)}=\bar{P}^{(n)} \in R_{1}$. Then from Lemma 2, remembering that $n$ is even,

$$
\begin{aligned}
& P^{(n+1)}=T P^{(n)}-(15+4 \sqrt{11}, 15-4 \sqrt{11}) \\
& =\left(\frac{1}{2}+\frac{15}{22} \sqrt{11}+\tau(-1)^{n} \sum_{1}^{\infty}(-\tau)^{-m} \alpha_{m+n-1}, \frac{1}{2}-\frac{15}{22} \sqrt{11}-\tau(-1)^{n} \sum_{1}^{\infty}(-\tau)^{1-m} \alpha_{n-m}\right) \\
& =\left(\frac{1}{2}+\frac{15}{22} \sqrt{11}-\alpha_{n}+(-1)^{n+1} \sum_{1}^{\infty}(-\tau)^{-m} \alpha_{m+n},\right. \\
& \left.\frac{1}{2}-\frac{15}{22} \sqrt{11}-\alpha_{n}-(-1)^{n+1} \sum_{1}^{\infty}(-\tau)^{1-m} \alpha_{n+1-m}\right) \\
& =\bar{P}^{(n+1)}
\end{aligned}
$$

and this argument can clearly be reversed. Similarly for the other regions. This proves the Lemma.

Lemma 8. Let $P$ be admissible, and write

$$
M_{n}^{ \pm}(\alpha)=M_{n}^{ \pm}=\left(\frac{7}{22} \sqrt{11} \pm \frac{1}{2}+\sum_{1}^{\infty}(-\tau)^{-m} \alpha_{m+n-1}\right)\left(\frac{7}{22} \sqrt{11} \mp \frac{1}{2}+\sum_{1}^{\infty}(-\tau)^{1-m} \alpha_{n-m}\right) .
$$

Then $M(f, P)=\operatorname{Min}\left\{M_{n}^{\star}\right\}$, taken over all $n$ and both signs.

It is clear that every $M_{n}^{ \pm}>0$, since both factors are positive.

By Theorem B, the set of values less than $\frac{19}{22}=M_{1}(f)$ taken by $f$ at points congruent to $P$ is the same as the set of values less than $\frac{19}{22}$ taken by $f$ at points $(x, y)$ congruent to some $P^{(n)}$ with

$$
y^{2}<\frac{19}{44},|y|<0.6572
$$

From this and $\left|x^{2}-11 y^{2}\right|<\frac{19}{22}$, we deduce also that

$$
x^{2}<\frac{247}{44},|x|<2.3694
$$


We must now find which hyperbolae we need to consider in each of the four regions. We write a suffix $n$ for the co-ordinates of $P^{(n)}$.

(i) $P^{(n)} \in \mathcal{R}_{1}$. Then from Lemma 3

$$
2 \lambda_{2}-1 \leq \xi_{n}-\eta_{n}=2 y_{n} \sqrt{11} \leq 2 \lambda_{1}-1,
$$

whence $0.2999<y_{n}<0.3192$; and by (12), $0.3714<x_{n}<0.6286$. Thus by (19) and (20) we need only consider $f\left(x_{n}-2, y_{n}\right), f\left(x_{n}-1, y_{n}\right), f\left(x_{n}, y_{n}\right)$ and $f\left(x_{n}+1, y_{n}\right)$. But

$$
\begin{aligned}
& \left|f\left(x_{n}-2, y_{n}\right)\right|=\left|\left(\xi_{n}-2\right)\left(\eta_{n}-2\right)\right| \geq\left(2-\lambda_{1}\right)\left(1+\lambda_{2}\right)>0.441 \times 2.494>M(f), \\
& \left|f\left(x_{n}+1, y_{n}\right)\right|=\left|\left(\xi_{n}+1\right)\left(\eta_{n}+1\right)\right| \geq\left(2-\lambda_{1}\right)\left(1+\lambda_{2}\right)>M(f),
\end{aligned}
$$

so that we may neglect these two; for the other two we have

$$
f\left(x_{n}-1, y_{n}\right)=M_{n}^{-}, f\left(x_{n}, y_{n}\right)=M_{n}^{+},
$$

since $n$ is even.

(ii) $P^{(n)} \in R_{2}$. By symmetry from the previous case wo need only consider

$$
f\left(x_{n}-1, y_{n}-1\right)=M_{n}^{+}, f\left(x_{n}, y_{n}-1\right)=M_{n}^{-} .
$$

(iii) $P^{(n)} \in R_{3}$. Then we have $\alpha_{n-1}=1, \alpha_{n-2}=\alpha_{n}=0$, so that

$$
\begin{aligned}
M_{n-1}^{-} & \leq\left(\frac{7}{22} V_{11}-\frac{1}{2}-\tau^{-1}+\frac{\tau^{-4}}{1-\tau^{-2}}\right)\left(\frac{7}{22} \sqrt{11}+\frac{1}{2}+\frac{\tau^{-2}}{1-\tau^{-2}}\right) \\
& <0.5053 \times 1.5580<0.79 .
\end{aligned}
$$

But since $P^{(n-1)} \in R_{1}$ or $R_{2}$, the earlier parts of the proof of this Lemma show that we have $M(f, P) \leq M_{n-1}^{-}<0.79$, so that in $\boldsymbol{R}_{3}$ we need consider only values less than 0.79 . We now show there are none such.

In $R_{3}$ we need consider, by (12), (19) and (20), only

$$
\begin{gathered}
f\left(x_{n}, y_{n}\right), f\left(x_{n}, y_{n}-1\right) ; f\left(x_{n} \pm 1, y_{n}\right), f\left(x_{n} \pm 1, y_{n}-1\right) \\
f\left(x_{n} \pm 2, y_{n}\right), f\left(x_{n} \pm 2, y_{n}-1\right) .
\end{gathered}
$$

By the symmetry of $\boldsymbol{R}_{3}$ we need consider only one function from each group. Now we find

$$
\begin{aligned}
\left|f\left(x_{n}, y_{n}\right)\right| & =\left|\xi_{n} \eta_{n}\right| \geq\left(\sqrt{11}-\lambda_{3}\right)^{2}>M(f) \\
\left|f\left(x_{n}-1, y_{n}\right)\right| & =\left|\left(\xi_{n}-1\right)\left(\eta_{n}-1\right)\right| \geq\left(\sqrt{11}-\lambda_{3}-1\right)\left(\sqrt{11}-\lambda_{3}+1\right)>M(f) \\
\left|f\left(x_{n}-2, y_{n}\right)\right| & =\left|\left(\xi_{n}-2\right)\left(\xi_{n}-2\right)\right| \geq\left(2-\lambda_{3}\right)\left(2+\sqrt{11}-\lambda_{3}\right) \\
& >0.2385 \times 3.5551>0.8478>0.79 .
\end{aligned}
$$


To conclude the proof, we need merely remark that if $P^{(n)} \in \mathcal{R}_{11}$ then $M_{n}^{+}=$ $=f\left(x_{n}, y_{n}-1\right), M_{n}^{-}=f\left(x_{n}+1, y_{n}-1\right)$; while if $P \in \boldsymbol{R}_{22}, M_{n}^{+}=f\left(x_{n}, y_{n}\right), M_{n}^{-}=f\left(x_{n}-1, y_{n}\right)$.

Lemmas 5 and 8 at last give us a sound algebraic basis on which to work. We have obtained in the course of the proof the further results:

Corollary 1. If the sequence $\left\{\alpha_{n}\right\}$ contains any elements $1, M(f, P)<0.79<M(f)$.

Corollary 2. In evaluating the Min in Lemma 8, we may confine ourselves to those $n$ for which $\alpha_{n-1}=0$.

It follows from the first of these that the first minimum corresponds to the sequence $\alpha_{n}=0$, as may be verified by direct calculation.

Now write $\bar{\alpha}_{n}=\alpha_{-n}$, so that the sequence $\left\{\bar{\alpha}_{n}\right\}$ is the reverse of the sequence $\left\{\alpha_{n}\right\}$. Then if $\alpha_{n-1}=0$,

$$
M_{n}^{+}(\alpha)=\left(\frac{7}{22} \sqrt{11}+\frac{1}{2}+\sum_{1}^{\infty}(-\tau)^{-m} \alpha_{m+n-1}\right)\left(\frac{7}{22} \sqrt{11}-\frac{1}{2}+\sum_{1}^{\infty}(-\tau)^{-m} \alpha_{n-m-1}\right)=M_{2-n}^{-}(\bar{\alpha}) ;
$$

so that by Lemma 8 and Corollary 2 we find the more symmetric form

$$
M(f, P)=\operatorname{Min}\left[M_{n}^{-}(\alpha), M_{n}^{-}(\bar{\alpha})\right],
$$

which we shall use henceforth.

Now we want a simple rule for comparing the values of two $M_{n}^{-}(\alpha)$; and since changing $n$ in $M_{n}^{-}(\alpha)$ is equivalent merely to starting the doubly infinite sequence $\left\{\alpha_{n}\right\}$ in a different place, it is enough to compare $M_{0}^{-}(\alpha), M_{0}^{-}(\beta)$.

Lemma 9. Let $\{\alpha\},\{\beta\}$ be two sequences satisfying the conditions of Lemma 5 . Let the terms be written, for comparison, in the order

$$
\begin{aligned}
& \alpha_{-1}, \alpha_{0}, \alpha_{-2}, \alpha_{1}, \alpha_{-3}, \alpha_{2}, \ldots \\
& \beta_{-1}, \beta_{0}, \beta_{-2}, \beta_{1}, \beta_{-3}, \beta_{2}, \ldots
\end{aligned}
$$

and let $\alpha_{\tau_{0}}, \beta_{r_{0}}$ be the first distinct pair. Then $M_{0}^{-}(\alpha)-M_{0}^{-}(\beta)$ has the sign of

Write

$$
(-1)^{r_{0}+1}\left(\alpha_{r_{0}}-\beta_{r_{0}}\right)
$$

so that

$$
\begin{aligned}
& \xi(\alpha)=\frac{7}{22} \sqrt{11}-\frac{1}{2}+\sum_{1}^{\infty}(-\tau)^{-n} \alpha_{n-1}, \\
& \dot{\eta}(\alpha)=\frac{7}{22} \sqrt{11}+\frac{1}{2}+\sum_{1}^{\infty}(-\tau)^{1-n} \alpha_{-n},
\end{aligned}
$$

$$
M^{-}(\alpha)-M^{-}(\beta)=\xi(\alpha) \eta(\alpha)-\xi(\beta) \eta(\beta)=\xi(\alpha)\{\eta(\alpha)-\eta(\beta)\}+\eta(\beta)\{\xi(\alpha)-\xi(\beta)\} .
$$

19-523804. Acta mathematica. 88. Imprimó le 16 décembre $195 \dot{2}$. 
It is easily seen (cf. the proof of Lamma 7 ) that for any sequence $\{\alpha\}$

$$
\frac{10}{33} \sqrt{11}-\frac{1}{2} \leq \xi(\alpha) \leq \frac{31}{66} \sqrt{11}-1, \quad \frac{10}{33} \sqrt{11}+\frac{1}{2} \leq \eta(\alpha) \leq \frac{31}{66} \sqrt{11}+1 .
$$

Let $r_{1} \geq 0, r_{2}<0$ be the values of $r$ nearest to 0 on each side for which $\alpha_{r} \neq \beta_{r}$, so that $r_{0}=r_{1}$ or $r_{2}$ according as $r_{1}+r_{2} \leq-2$ or $\geq-1$. The first non-zero term in $\xi(\alpha-\beta)$ is given by $n=r_{1}+1$, and the first non-zero term in $\eta(\alpha-\beta)$ by $n=-r_{2}$. Hence, since

$$
1-\tau^{-1}-\tau^{-2}-\cdots=\frac{9-\sqrt{11}}{6}, \quad 1+\tau^{-1}+\tau^{-2}+\cdots=\frac{3+\sqrt{11}}{6},
$$

we deduce that

$$
\left.\begin{array}{l}
\frac{9-\sqrt{11}}{6} \leq(-\tau)^{r_{1}+1}\left(\alpha_{r_{1}}-\beta_{r_{1}}\right)\left(\xi(\alpha)-\xi(\beta) \leq \frac{3+\sqrt{11}}{6},\right. \\
\frac{9-\sqrt{11}}{6} \leq(-\tau)^{-1-r_{2}}\left(\alpha_{r_{2}}-\beta_{r_{2}}\right)(\eta(\alpha)-\eta(\beta)) \leq \frac{3+\sqrt{11}}{6} .
\end{array}\right\}
$$

If $r_{0}=r_{1}$, we have $r_{1}+r_{2} \leq-2$ and it is easily verified from (22) and (23) that

$$
\mid \xi(\alpha)(\eta(\alpha)-\eta(\beta)|<| \eta(\beta)(\xi(\alpha)-\xi(\beta)) \mid,
$$

and so $M_{0}^{-}(\alpha)-M_{0}^{-}(\beta)$ has the sign of $\xi(\alpha)-\xi(\beta)$, i.e. of $(-\tau)^{r_{0}+1}\left(\alpha_{r_{0}}-\beta_{r_{0}}\right)$.

Similarly, if $r_{0}=r_{2}$, we have $r_{1}+r_{2} \geq-1$ and

$$
|\xi(\alpha)(\eta(\alpha)-\eta(\beta))|>|\eta(\beta)(\xi(\alpha)-\xi(\beta))|,
$$

and so $M_{0}^{-}(\alpha)-M_{0}^{-}(\beta)$ has the sign of $\eta(\alpha)-\eta(\beta)$, i.e. of $(-\tau)^{r_{0}+1}\left(\alpha_{r_{0}}-\beta_{r_{0}}\right)$.

We must next consider sequences $\{\alpha\}$. To save space, we write []$_{k}$ to indicate that the set of elements inside the square brackets is to be repeated $k$ times. In the special case $k=\infty$, the side(s) of the brackets on which $\infty$ lies will represent in which direction(s) the infinite repetition happens.

After Corollary 1 of Lemma 8, we may assume that not every $\alpha_{n}$ is 0 . We look for those sequences $\{\alpha\}$ giving a larger $\operatorname{Min}\left[M_{n}^{-}(\alpha), M_{n}^{-}(\bar{\alpha})\right]$ than the sequence

$$
\left\{\alpha^{(\infty)}\right\}: \quad \infty[100] 100001[001]_{\infty} \text {. }
$$

This sequence is symmetric, and it is easily seen from Lemma 9 that the least $M_{n}^{-}\left(\alpha^{(\infty)}\right)$ occurs when $\alpha_{n}^{(\infty)}=1$, preceded by four elements 0 . Thus for the corresponding point, taking $n=0$ for convenience, 


$$
\begin{aligned}
M(f, P)=M_{0}^{-} & =\left(\frac{7}{22} \sqrt{11}-\frac{1}{2}-\tau^{-1}+\tau^{-4}-\tau^{-7}+\cdots\right)\left(\frac{7}{22} \sqrt{11}+\frac{1}{2}+\tau^{-4}-\tau^{-7}+\cdots\right) \\
& =\left(\frac{7}{22} \sqrt{11}-\frac{1}{2}-\frac{\tau^{2}}{\tau^{3}+1}\right)\left(\frac{7}{22} \sqrt{11}+\frac{1}{2}+\frac{1}{\tau\left(\tau^{3}+1\right)}\right) \\
& =\frac{5(3711 \sqrt{11}-11684)}{3971},
\end{aligned}
$$

the value we have asserted for $M_{\infty}(f)$. We shall call a sequence strongly admissible if it has an $M(f, P)$ not less than this, i.e. if $M_{n}^{-}(\alpha) \geq M_{0}^{-}\left(\alpha^{(\infty)}\right)$ for all $n$.

We shall now obtain certain conditions on strongly admissible sequences. In each case we use Lemma 9 to show that $M_{0}^{-}\left(\alpha^{(\infty)}\right)>M_{0}^{-}(\alpha)$, when $\{\alpha\}$ satisfies some condition and the starting point of it is suitably chosen.

By taking in each case $\alpha_{0}$ as the element in heavy type, we find that no strongly admissible sequence can contain any of:

$$
\ldots 101 \ldots, \ldots 1000100 \ldots, \ldots 0001000 \ldots, \ldots 00001001 \ldots
$$

or the reversed subsequences. It follows from this that every strongly admissible sequence is made up of blocks of 001 and 00001 , and that we cannot have two conseoutive blocks of the latter sort.

Now suppose that a strongly admissible sequence contains

$$
\text { ... } 00001[001]_{m} 00001[001]_{n} 00001 \ldots
$$

and, taking $\alpha_{0}$ to be the 1 in heavy type, compare $M_{0}^{-}(\alpha)$ with $M_{0}^{-}\left(\alpha^{(\infty)}\right)$. The first terms which are different, before and after $\alpha_{0}$, are $\alpha_{3 n+3}=0$ and $\alpha_{-3 n-8}=0$. Thus from Lemma 9, for the sequence to be strongly admissible we must have

or

$$
\begin{aligned}
& n \leq m+1, \quad 3 n+3 \text { even (and so } n \text { odd) } \\
& n \geq m+2, \quad 3 m+8 \text { even (and so } m \text { even). }
\end{aligned}
$$

By considering the reversed sequence, we must have in the same way

or

$$
\begin{aligned}
& m \leq n+1, \quad 3 m+3 \text { even (and so } m \text { odd) } \\
& m \geq n+2, \quad 3 n+8 \text { even (and so } n \text { even). }
\end{aligned}
$$

Comparing the parity conditions, since we cannot have both $m \geq n+2$ and $n \geq m+1$, we must have $n \leq m+1, m \leq n+1, m$ and $n$ both odd, whence $m=n$.

Thus the only possible strongly admissible sequences are

$$
\infty_{\infty}[0]_{\infty}, \infty[001]_{\infty}, \infty[100] 100001[001]_{\infty}, \infty\left[00001[001]_{2 k+1}\right]_{\infty} \quad(k=0,1,2, \ldots) .
$$

It only remains to find $M(f, P)$ and a generating point for each of these sequences. 
The sequence $\infty_{\infty}[0]_{\infty}$ is already known to be that given by (1) and (6) - the first minimum.

For the sequence ${ }_{\infty}[001]_{\infty}$ we have at once

$$
\begin{aligned}
M(t, P) & =\left(\frac{7}{22} \sqrt{11}-\frac{1}{2}-\tau^{-1}+\tau^{-4}-\tau^{-7}+\cdots\right)\left(\frac{7}{22} \sqrt{11}+\frac{1}{2}+\tau^{-2}-\tau^{-5}+\cdots\right) \\
& =\left(\frac{7}{22} \sqrt{11}-\frac{1}{2}-\frac{\tau^{2}}{\tau^{3}+1}\right)\left(\frac{7}{22} \sqrt{11}+\frac{1}{2}+\frac{\tau}{\tau^{3}+1}\right)=\frac{3125}{3971}
\end{aligned}
$$

The generating point, in $(\xi, \eta)$ co-ordinates, is $\left(\frac{7}{22} \sqrt{11}+\frac{1}{2}-\frac{\tau^{2}}{\tau^{3}+1}, \frac{1}{2}-\frac{7}{22} \sqrt{11}-\frac{\tau}{\tau^{3}+1}\right)$, which reduces, in $(x, y)$ co-ordinates, to $\left(\frac{9}{19}, \frac{65}{209}\right)$. The transforms by $T$ of this are $\left(0, \frac{98}{209}\right)$ and $\left(\frac{10}{19}, \frac{65}{209}\right)$, with their images in the origin.

For the sequence $\infty[100] 100001[001]_{\infty}$ we have already found $M(f, P)$. The point series is generated by $\left(\frac{7}{22} \sqrt{11}+\frac{1}{2}-\frac{\tau^{2}}{\tau^{3}+1}, \frac{1}{2}-\frac{7}{22} \sqrt{11}-\frac{\tau^{-1}}{\tau^{3}+1}\right)$ in $(\xi, \eta)$ co-ordinates, which reduces, in $(x, y)$ co-ordinates, to $\left(\frac{3 \sqrt{11}-9}{2}, \frac{189 \sqrt{11}-497}{418}\right)$.

It remains to study the sequence

$$
{ }_{\infty}\left[00001[001]_{2 k+1}\right]_{\infty} .
$$

It follows from Lemma, 9 that $M(f, P)=M_{0}^{-}(\alpha)$, where $\alpha_{0}=1$ is preceded by four elements 0 . This gives, remembering the definition of $\mu_{k}$ in (4),

$$
\begin{aligned}
& \sum_{1}^{\infty}(-\tau)^{-n} \alpha_{n-1}=\left(-\tau^{-1}+\tau^{-4}-\cdots+\tau^{-6 k-4}\right)+\left(-\tau^{-6 k-9}+\cdots+\tau^{-12 k-12}\right)+\cdots \\
&=\left(-\tau^{-1}+\tau^{-4}-\cdots+\tau^{-6 k-4}\right)\left(1+\tau^{-6 k-8}+\cdots\right)=-\frac{\mu_{k}}{1+\tau^{-3}} ;
\end{aligned}
$$

and similarly

$$
\sum_{1}^{\infty}(-\tau)^{1-n} \alpha_{-n}=\frac{\mu_{k}}{1+\tau^{3}} .
$$

Thus

$$
\begin{aligned}
M(f, P) & =M_{0}^{-}(\alpha)=\left(\frac{7}{22} \sqrt{11}-\frac{1}{2}-\frac{\mu_{k}}{1+\tau^{-3}}\right)\left(\frac{7}{22} \sqrt{11}+\frac{1}{2}+\frac{\mu_{k}}{1+\tau^{3}}\right) \\
& =\frac{19}{22}-\frac{325}{209} \mu_{k}-\frac{1}{7942} \mu_{k}^{2} .
\end{aligned}
$$


The corresponding point is, in $(\xi, \eta)$ co-ordinates,

$$
\left(\frac{7}{22} \sqrt{11}+\frac{1}{2}-\frac{\mu_{k}}{1+\tau^{-3}}, \frac{1}{2}-\frac{7}{22} \sqrt{11}-\frac{\mu_{k}}{1+\tau^{3}}\right),
$$

which simplifies, in $(x, y)$ coordinates, to $\left(\frac{1-\mu_{k}}{2}, \frac{133-63 \mu_{k}}{418}\right)$.

Thus the possible strongly admissible sequences have the values of $M(f, P)$ given in (1), (2), (3), (5), and are obtained from the corresponding point series specified by (6), (7), (8), (9). All that remains is to arrange these minima in decreasing order. This we could do from Lemma 9 ; but it is simpler to argue as follows.

We can verify directly that $\frac{19}{22}>\frac{3125}{2971}>\frac{19}{22}-\frac{325}{209} \mu_{0}-\frac{1}{7942} \mu_{0}^{2}$. Moreover

$$
\frac{5(3711 \sqrt{11}-11684)}{3971}=\frac{19}{22}-\frac{325}{209} \tau^{-1}-\frac{1}{7942} \tau^{-2} \text {. }
$$

All the results we want now follow from the fact that $\mu_{k}$ is monotone increasing and tends to $\tau^{-1}$.

It only remains to prove the last sentence of the theorem. It follows from the proof of Lemma 9 that if $\alpha_{n}=\beta_{n}$ for all $|n|<n_{0}(\varepsilon)$, then $\left|M_{0}^{-}(\alpha)-M_{0}^{-}(\beta)\right|<\varepsilon$. From this, we deduce that all sequences consisting of blocks 00001 and 001 , with the 00001 all very far apart, have $M(f, P)$ very near $M_{\infty}(f)$ - and less, by the main result of the theorem. The number of such sequences has the cardinal number of the continuum, and there are an infinity of them which are periodic and so correspond to rational points.

This concludes the proof of the Theorem.

2. Throughout this section we write

$$
\begin{gathered}
f(x, y)=x^{2}+3 x y-y^{2}, \quad \xi=x+\frac{3+\sqrt{13}}{2} y, \quad \eta=x+\frac{3-\sqrt{13}}{2} y, \\
\tau=\frac{3+\sqrt{13}}{2}, \quad \tau^{\prime}=\frac{3-\sqrt{13}}{2}, \quad T=\left(\begin{array}{ll}
0 & 1 \\
1 & 3
\end{array}\right)
\end{gathered}
$$

so that $T$ is the fundamental automorph of $f(x, y)=\xi \eta$, and $\tau$ is the fundamental unit of the field $k(\sqrt{13})$, with $\tau \tau^{\prime}=-1$.

Owing to the existence of the trivial autamorphs $\left(\begin{array}{cc}0 & \pm 1 \\ \mp 1 & 0\end{array}\right)$ of $f(x, y)$, any point $(x, y)$ has not one conjugate as in the previous section but three: $(-x,-y),(y,-x)$ and $(-y, x)$. However, a point series may have $0,1,2$ or 3 distinct conjugate 
point series, and if they are finite they need not all be of the same length, as we can see from the example

$$
\left(\frac{1}{3}, \frac{1}{3}\right) ;\left(-\frac{1}{3},-\frac{1}{3}\right) ;\left(\frac{1}{3},-\frac{1}{3}\right) \rightarrow\left(-\frac{1}{3}, \frac{1}{3}\right) .
$$

We know already that $f(x, y)$ cannot, in its chain of minima, behave exactly as $x^{2}-11 y^{2}$, for Theorem 7 of our previous paper shows that $M_{1}(f)$ is taken for two distinct types of point series, one rational and the other irrational; but if we ignore this distinction we could state a Theorem very similar to Theorem 1 above, whose proof, as we shall see, would be relatively simple. However, the analogy breaks down when we come to the last sentence of Theorem 1, for we find that there are only an enumerable infinity of values of $M(f, P)$, each corresponding to one point series and its conjugates, in a neighbourhood of $M_{\infty}(f)$. Thus we may continue the investigation below $M_{\infty}(f)$, obtaining the more complete result of Theorem 2 below.

Theorem 2. With the notation above, let us write

$$
M_{1}=\frac{1}{3}, \quad M_{2}=\frac{4}{13}, \quad M^{\prime}=\frac{26-4 \sqrt{13}}{39}, \quad M^{\mathrm{IV}}=\frac{51 \sqrt{13}-177}{26}=\frac{51 \tau-165}{13} .
$$

Then there are constants $M_{k}^{\prime}(k>0), M_{k}^{\prime \prime}(k>0), M_{k, h}^{\prime \prime \prime}(h, k>0), M_{k}^{\prime \prime \prime}(k>0), M_{1}, M_{2}, M_{k}^{\prime \prime}$ alone being rational, satistying

$$
\begin{aligned}
& M_{1}>M_{2}>M_{2}^{\prime}>M_{4}^{\prime}>\cdots>M^{\prime}>\cdots>M_{3}^{\prime}>M_{1}^{\prime} \\
> & M_{1}^{\prime \prime}>M_{1}^{\prime \prime \prime}>\cdots>M_{1,3}^{\prime \prime \prime}>M_{1,2}^{\prime \prime \prime}>M_{, 1}^{\prime \prime \prime} \\
> & M_{2}^{\prime \prime}>M_{2}^{\prime \prime \prime}>\cdots>M_{2,3}^{\prime \prime \prime}>M_{2,2}^{\prime \prime \prime}>M_{2,1}^{\prime \prime \prime} \\
> & \cdots \\
> & M^{\mathrm{IV}}
\end{aligned}
$$

with limit points

$$
M^{\prime}=\lim _{k \rightarrow \infty} M_{k}^{\prime}, \quad \boldsymbol{M}_{k}^{\prime \prime \prime}=\lim _{h \rightarrow \infty} \boldsymbol{M}_{k, h}^{\prime \prime \prime}, \quad \boldsymbol{M}^{\mathrm{Iv}}=\lim _{k \rightarrow \infty} \boldsymbol{M}_{k}^{\prime \prime}=\lim _{k \rightarrow \infty} \boldsymbol{M}_{k}^{\prime \prime \prime},
$$

such that the set of values of $M(f, P)$ not less than $M^{\mathrm{TV}}$ is precisely the set of numbers in (26). Any such value of $M(f, P)$ is taken only at the points of one point series and its conjugates - finite if $M(f, P)$ is rational and infinite otherwise; except that $M_{1}$ is taken both at four rational points and at the points of four infinite conjugate point series. All these values of $M(f, P)$ are attained except $M_{1}$. 
On the other hand, for any fixed $\varepsilon>0$ the set of values of $M^{\mathrm{IV}}>M(f, P)>M^{\mathrm{IV}}-\varepsilon$ has the cardinal number of the continuum, and contains an infinity of values taken at rational points.

The precise values of $M_{k}^{\prime}, M_{k}^{\prime \prime}, M_{k}^{\prime \prime \prime}, M_{k, h}^{\prime \prime \prime}$ will be obtained below (equations (34), (36), (39), (41) respectively) together with the points that generate the corresponding point series.

The proof of Theorem 2 is basically similar to that of Theorem 1, and we shall set it out similarly as a chain of lemmas. To save space, where the proof of a lemma is sufficiently similar to that of a lemma in $\S 1$, we shall omit it, giving instead a reference.

Lemma 10. If $M(f, P)>\frac{1}{4}$, then $P$ lies $(\bmod 1)$ in the region

$$
R: \quad \frac{1+\tau}{3} \leq \xi \leq \frac{2+2 \tau}{3}, \quad \frac{7-2 \tau}{3} \leq \eta \leq \frac{5-\tau}{3},
$$

and the same is true for $T^{n} P$ for every integer $n$.

Moreover, if $P \in \mathcal{R}$, then

and

$$
\text { TPER+(0,1) or } T P \in R+(0,2) \text {, }
$$

$$
T^{-1} P \in R+(-1,0) \text { or } T^{-1} P \in \mathcal{R}+(-2,0) \text {. }
$$

This is the analogue of Lemmas 1,2 and 3.

In the unit square $0 \leq x, y \leq 1$ we have

$$
0 \leq \xi \leq 1+\tau, \quad 3-\tau \leq \eta \leq 1 .
$$

Now in the region $0 \leq \xi \leq 1,0 \leq \eta \leq 1$ we have $|f(x, y)|=|\xi \eta| \leq \frac{1}{4}$ or $|f(x-1, y)|=$ $=|(1-\xi)(1-\eta)| \leq \frac{1}{4}$, according as $\xi+\eta \leq 1$ or $\xi+\eta \geq 1$; thus we may neglect this and the three conjugate regions,

$$
\begin{array}{ll}
\tau \leq \xi \leq \tau+1, & 3-\tau \leq \eta \leq 4-\tau \\
0 \leq \xi \leq \tau, & 3-\tau \leq \eta \leq 0 \\
1 \leq \xi \leq \tau+1, & 4-\tau \leq \eta \leq 1
\end{array}
$$

and we have only to consider

$$
\boldsymbol{R}^{\prime}: 1<\xi<\tau, \quad 0<\eta<4-\tau .
$$


Now since $y \sqrt{13}=\xi-\eta, x \sqrt{13}=\tau \eta-\tau^{\prime} \xi=\tau \eta+(\tau-3) \xi$, we have in $R^{\prime}$

$$
\tau-3<y \sqrt{13}<\tau, \quad \tau-3<x \sqrt{13}<\tau .
$$

But $T R^{\prime}$ is $\tau<\xi<\tau^{2}=3 \tau+1, \tau^{\prime}(4-\tau)=13-4 \tau<\eta<0$, so that

$$
\tau<y \sqrt{13}<7 \tau-12, \quad \tau-3<x \sqrt{13}<\tau .
$$

Comparison of (27) and (28) shows that the transformation laws stated in Lemma 10 for $R$ hold already for $\boldsymbol{R}^{\prime}$; now an argument analogous to that of Lemma 3 shows that we can reduce $\mathcal{R}^{\prime}$ to $R$, in virtue of

$$
\tau\left(\frac{1+\tau}{3}\right)=\frac{1+\tau}{3}+\tau
$$

and the three similar equations. This proves the Lemma.

As in the previous section, we define $P$ as admissible if, for every integer $n$, $T^{n} P$ lies in $\mathcal{R}(\bmod 1)$. Thus by Lemma 10 all points with $M(f, P)>\frac{1}{4}$ are admissible. For any admissible $P$ and any integer $n$, we define $P^{(n)}$ as the point of $\boldsymbol{R}$ congruent to $T^{n} P(\bmod 1)$.

We now subdivide $\mathcal{R}$. We define the four regions $\boldsymbol{R}_{i}(i=1,2,3,4)$ as the set of points $P \in R$ for which

$$
\begin{array}{lll}
\mathcal{R}_{1}: & T P \in R+(0,1), & T^{-1} P \in \mathcal{R}+(-1,0) ; \\
\mathcal{R}_{2}: & T P \in R+(0,1), & T^{-1} P \in \mathcal{R}+(-2,0) ; \\
\mathcal{R}_{3}: & T P \in \mathcal{R}+(0,2), & T^{-1} P \in \mathcal{R}+(-1,0) ; \\
\mathcal{R}_{4}: & T P \in R+(0,2), & T^{-1} P \in R+(-2,0) .
\end{array}
$$

Transforming the equations in each line by $T^{-1}, T$ respectively, and comparing, we find that for admissible points $P$,

$$
\begin{aligned}
& \text { if } P \in R_{1} \text { then } P^{(1)} \in R_{1} \text { or } R_{3} \text { and } P^{(-1)} \in R_{1} \text { or } R_{2} \text {; } \\
& \text { if } P \in R_{2} \text { then } P^{(1)} \in R_{1} \text { or } R_{3} \text { and } P^{(-1)} \in R_{3} \text { or } R_{4} \text {; } \\
& \text { if } P \in R_{3} \text { then } P^{(1)} \in R_{2} \text { or } R_{4} \text { and } P^{(-1)} \in R_{1} \text { or } R_{2} \text {; } \\
& \text { if } P \in R_{4} \text { then } P^{(1)} \in R_{2} \text { or } R_{4} \text { and } P^{(-1)} \in R_{3} \text { or } R_{4} \text {; }
\end{aligned}
$$

We now define, for all admissible $P$,

$$
\alpha_{n}=\alpha_{n}(P)=\left\{\begin{array}{lll}
1 & \text { if } P^{(n)} \in \mathcal{R}_{3} & \text { or } \mathcal{R}_{4}, \\
0 & \text { if } P^{(n)} \in \mathcal{R}_{1} & \text { or } \mathcal{R}_{3} .
\end{array}\right.
$$


Lemma 11. Given any doubly-infinite sequence of elements 0,1 , there is an admissible point $P$ which has this sequence for its $\left\{\alpha_{n}\right\}$, and $P$ is uniquely defined $(\bmod 1)$ by the sequence. Moreover, using $(\xi, \eta)$-coordinates, $P^{(n)}$ is given by

$$
\left(\frac{1+\tau}{3}+\sum_{m=0}^{\infty} \tau^{-m} \alpha_{m+n}, \quad \frac{4-\tau}{3}-\sum_{m=1}^{\infty}\left(-\tau^{-1}\right)^{m} \alpha_{n-m}\right) .
$$

The proof of this is similar to the proofs of Lemmas 5 and 7 , the existence and uniqueness corresponding to Lemma 5 and the actual formula to Lemma 7 . If we denote the coordinates of $P^{(n)}$ by a suffix $n$, the actual formula may be verified from the equations

$$
\xi_{n+1}=\tau \xi_{n}-\tau\left(1+\alpha_{n}\right), \quad \eta_{n+1}=\tau^{-1}\left(1+\alpha_{n}\right)-\tau^{-1} \eta_{n}
$$

We note that the conditions for $P^{(0)}$ to lie in any particular $\boldsymbol{R}_{\boldsymbol{i}}$ are as follows

$$
\begin{array}{llll}
\mathcal{R}_{1}: & \alpha_{0}=0, \alpha_{-1}=0 ; & \mathcal{R}_{2}: & \alpha_{0}=0, \alpha_{-1}=1 ; \\
\mathcal{R}_{3}: & \alpha_{0}=1, \alpha_{-1}=0 ; & \mathcal{R}_{4}: & \alpha_{0}=1, \alpha_{-1}=1 .
\end{array}
$$

Now the three conjugate points to $(\xi, \eta)$ are respectively $\left(1+\tau-\xi, 1-\tau^{-1}-\eta\right)$, $\left(1+\tau \eta, 1-\tau^{-1} \xi\right)$ and $\left(\tau-\tau \eta, \tau^{-1} \xi-\tau^{-1}\right)$; and we may now deduce from Lemma 11, with a little algebraic manipulation, that the three points conjugate to $\left\{\alpha_{n}\right\}$ may be obtained from it by the operations

(i) write 1,0 for 0,1 respectively;

(ii) write 1,0 for 0,1 respectively when $n$ is even, and then interchange $\alpha_{n}$ with $\alpha_{-n-1}$;

(iii) write 1,0 for 0,1 respectively when $n$ is odd, and then interchange $\alpha_{n}$ with $\alpha_{-n-1}$.

We have next to find which hyperbolae we need consider; in other words, to obtain an equivalent result to Lemma 8 .

Lemma 12. If $P$ is admissible and $P^{(n)}$ is $\left(\xi_{n}, \eta_{n}\right)$, then

$$
M(f, P)=\operatorname{Min}\left|f_{n}(\alpha)\right|
$$

taken over all $n$, where $f_{n}(\alpha)=\left(\xi_{n}-\alpha_{n-1}-\tau \alpha_{n}\right)\left(\eta_{n}-\alpha_{n-1}+\tau^{-1} \alpha_{n}\right)$.

In other words, if $P^{(n)}$ lies in $\boldsymbol{R}_{1}, \boldsymbol{R}_{2}, \boldsymbol{R}_{3}, \boldsymbol{R}_{\mathbf{4}}$ respectively, then we need only consider $f(x, y), f(x-1, y), f(x, y-1)$ or $f(x-1, y-1)$ respectively. 
It is easily verified that Lemma 12 holds for the known set of points at which $M(f, P)=M_{1}(f)=\frac{1}{3}$; a result which follows at once from $f\left(\frac{1}{3}, \frac{1}{3}\right)=\frac{1}{3}$ and the conjugate results. Thus in Theorem $\mathrm{B}$ we can take $K=\frac{1}{3}$, so that the bound we obtain is $|y|<\frac{1}{3}$, and so $\left|x+\frac{3}{2} y\right|<\frac{5}{6},|x|<\frac{4}{3}$.

Now in $R$ we have $x+\tau y=\xi \geq \frac{1+\tau}{3}$, so that if $y<\frac{1}{3}, x>\frac{1}{3}$; and $x-\tau^{-1} y=$ $=\eta \leq \frac{2-\tau^{-1}}{3}$, so that if $y<\frac{1}{3}, x<\frac{2}{3}$. Using also the similar results when $y>\frac{2}{3}$, we find that we need only consider $f(x, y), f(x-1, y), f(x, y-1), f(x-1, y-1)$. We can deduce either from Lemma 11 or from the definition that in $R_{1}$ and $R_{2}, \xi \leq \frac{2 \tau+1}{3}$, so that

$$
y \sqrt{13}=\xi-\eta \leq \frac{4 \tau-8}{3}=\frac{2}{3}(\sqrt{13}-1), \quad y<\frac{2}{3}
$$

so that in $\boldsymbol{R}_{1}$ and $\boldsymbol{R}_{2}$ we need only consider $f(x, y), f(x-1, y)$.

In $\boldsymbol{R}_{2}$ we have $\xi \geq \frac{1+\tau}{3}, \eta \geq \frac{\tau-2}{3}$, so that $|f(x, y)|=\xi \eta \geq \frac{2 \tau-1}{9}>\frac{1}{3}$. If $P^{(n)} \in \boldsymbol{R}_{1}$ we have to separate cases. First if $P^{(n+1)} \in \boldsymbol{R}_{1}$, then since $x_{n+1}=y_{n}, y_{n+1}=x_{n}+3 y_{n}-1$, we have

$$
f\left(x_{n}-1, y_{n}\right)=-f\left(y_{n}, x_{n}+3 y_{n}-1\right)=-f\left(x_{n+1}, y_{n+1}\right),
$$

a value which is already being considered. Second if $P^{(n+1)} \in R_{3}$, then $\xi_{n} \geq \frac{4 \tau-8}{3}$, so that

$$
\left|f\left(x_{n}-1, y_{n}\right)\right|=\left(\xi_{n}-1\right)\left(1-\eta_{n}\right) \geq \frac{4 \tau-11}{3} \times \frac{4 \tau-11}{3}=\frac{137-40 \tau}{9}>\frac{1}{3} .
$$

Thus in neither case need we consider $f(x-1, y)$. With the similar results for $\boldsymbol{R}_{\mathbf{3}}$ and $\boldsymbol{R}_{\mathbf{4}}$, this proves the Lemma.

We have now to find a rule for comparing two values of $f_{n}(\alpha)$; or, which comes to the same thing, for comparing $f_{0}(\alpha)$ and $f_{0}(\beta)$ for any two sequences $\{\alpha\}$, $\{\beta\}$. It will be seen that replacing $\{\alpha\}$ by any of its three conjugate sequences leaves $\left|f_{0}(\alpha)\right|$ unchanged; so we may assume for our purpose that $\alpha_{0}=\alpha_{-1}=0$, $\beta_{0}=\beta_{-1}=0$, in which case $f_{0}(\alpha)>0, f_{0}(\beta)>0$.

Lemma 13. Lथ $\beta_{-2}=1, \beta_{-1}=\beta_{0}=\beta_{1}=0$, and let $\alpha_{-1}=\alpha_{0}=0$. Then either of the two following sets of conditions 


$$
\begin{aligned}
& \text { (i) } \alpha_{-2}=0, \\
& \text { (ii) } \alpha_{-3}=\alpha_{-2}=\alpha_{1}=1,
\end{aligned}
$$

is sufficient to ensure that $f_{0}(\alpha)>f_{0}(\beta)>0$.

In fact we have

$$
f_{0}(\beta) \leq\left(\frac{1+\tau}{3}+\frac{\tau^{-2}}{1-\tau^{-1}}\right)\left(\frac{4-\tau}{3}-\tau^{-2}+\frac{\tau^{-3}}{1-\tau^{-2}}\right)=\frac{8-\tau}{3} \times \frac{5 \tau-16}{3}=\frac{41 \tau-133}{9}
$$

Also in case (i)

$$
f_{0}(\alpha) \geq\left(\frac{1+\tau}{3}\right)\left(\frac{4-\tau}{3}-\frac{\tau^{-4}}{1-\tau^{-2}}\right)=\frac{1+\tau}{3} \times \frac{37-11 \tau}{9}=\frac{26-7 \tau}{9}>f_{0}(\beta)
$$

since $\tau<\frac{159}{48}$; and in case (ii)

$$
f_{0}(\alpha) \geq\left(\frac{1+\tau}{3}+\tau^{-1}\right)\left(\frac{4-\tau}{3}-\tau^{-2}+\tau^{-3}-\frac{\tau^{-4}}{1-\tau^{-2}}\right)=\frac{4 \tau-8}{3} \times \frac{28 \tau-92}{3}=\frac{848-256 \tau}{9}>f_{0}(\beta)
$$

since $\tau<\frac{981}{297}$.

As in the previous section the element $\alpha_{0}$ of a sequence $\{\alpha\}$ will be printed in heavy type. The comparison sequence $\{\beta\}$ in Lemma 13 is . . 1000 . ., and its three conjugates are, by the rules above, ..0111 .., ...1011 ., and . . $0100 \ldots$. The two possibilities for $\{\alpha\}$ not covered by the Lemma are . . 1000 . . and ...01001 ... In the latter case we have

$$
\left|f_{-1}(\alpha)\right|=\left|\left(\frac{1+\tau}{3}+\sum_{0}^{\infty} \tau^{-n} \alpha_{n-1}-1\right)\left(\frac{4-\tau}{3}-\sum_{1}^{\infty}\left(-\tau^{-1}\right)^{n} \alpha_{-n-1}-1\right)\right|=\left|f_{0}(\alpha)\right|,
$$

since $\tau\left(\frac{\tau-2}{3}\right)=\frac{\tau+1}{3}$ and $\tau^{-1}\left(\frac{1-\tau}{3}\right)=-\frac{4-\tau}{3}$. But if $P^{(0)}$ is $\left\{\alpha_{n}\right\}, P^{(-1)}$ is $\ldots 01001 \ldots$ which is a conjugate of $\ldots 1000 \ldots$ Hence we deduce

Corollary 1. If $\left\{\alpha_{n}\right\}$ contains none of the subsequences $1000,0111,1011,0100$, then $M(f, P)$ has a higher value than $\frac{41 \tau-133}{9}>\frac{1}{4}$; otherwise not.

Corollary 2. If $\left\{\alpha_{n}\right\}$ contains at least one of the subsequences 1000, 0111, 1011, 0100 , then in Lemma 12 we need only take the minimum over those values of $n$ for which $\alpha_{n}$ is the third term of one of these subsequences. 
Obviously our first concern must be with those sequences that satisfy the conditions of Corollary 1; but we shall find that it is also necessary to consider other sequences, and so we next set ourselves to find selection rules analogous to Lemma 9 . In this case, the selection rules are not complete (and it is in fact difficult to make them so); but they are sufficient for our purpose.

Lemma 14. Let $\{\alpha\},\{\beta\}$ be two sequences both containing . . 1000 . ; and let $-n_{1}, n_{2}$ be the greatest negative and least positive values of $n$ for which $\alpha_{n} \neq \beta_{n}$. Then

(i) if $n_{1} \leq n_{2}+1, f_{0}(\alpha)-f_{0}(\beta)$ has the sign of $(-)^{n_{1}-1}\left(\alpha_{-n_{1}}-\beta_{-n_{1}}\right)$;

(ii) if $n_{1} \geq n_{2}+3, f_{0}(\alpha)-f_{0}(\beta)$ has the sign of $\left(\alpha_{n_{2}}-\beta_{n_{2}}\right)$;

(iii) if $(-)^{n_{1}-1}\left(\alpha_{-n_{1}}-\beta_{-n_{1}}\right)=1$ and $\alpha_{n_{2}}-\beta_{n_{2}}=1, f_{0}(\alpha)>f_{0}(\beta)$.

In the obvious notation, $f_{0}(\alpha)=\xi_{0}(\alpha) \eta_{0}(\alpha)$. Then

$$
f_{0}(\alpha)-f_{0}(\beta)=\xi_{0}(\alpha)\left\{\eta_{0}(\alpha)-\eta_{0}(\beta)\right\}+\eta_{0}(\beta)\left\{\xi_{0}(\alpha)-\xi_{0}(\beta)\right\},
$$

and in this we must find estimates for the four expressions on the right. In fact

$$
\frac{\tau+1}{3} \leq \xi_{0}(\alpha) \leq \frac{8-\tau}{3}, \frac{7-2 \tau}{3} \leq \eta_{0}(\beta) \leq \frac{5 \tau-16}{3}
$$

and

$$
\begin{aligned}
& \tau^{-n_{2}}\left(1-\frac{1}{\tau-1}\right) \leq\left|\xi_{0}(\alpha)-\xi_{0}(\beta)\right| \leq \tau^{-n_{2}}\left(1+\frac{1}{\tau-1}\right), \\
& \tau^{-n_{1}}\left(1-\frac{1}{\tau-1}\right) \leq\left|\eta_{0}(\alpha)-\eta_{0}(\beta)\right| \leq \tau^{-n_{1}}\left(1+\frac{1}{\tau-1}\right) ;
\end{aligned}
$$

from which we obtain

$$
\begin{gathered}
\frac{7 \tau+2}{3} \leq \frac{\xi_{0}(\alpha)}{\eta_{0}(\beta)} \leq 3 \tau+2 \\
7-2 \tau \leq \tau^{n_{2}-n_{1}}\left|\frac{\xi_{0}(\alpha)-\xi_{0}(\beta)}{\eta_{0}(\alpha)-\eta_{0}(\beta)}\right| \leq \frac{2 \tau+1}{3} .
\end{gathered}
$$

If $n_{1} \leq n_{2}+1$ we have $\left|\xi_{0}(\alpha)\left\{\eta_{0}(\alpha)-\eta_{0}(\beta)\right\}\right| \geq\left|\eta_{0}(\beta)\left\{\xi_{0}(\alpha)-\xi_{0}(\beta)\right\}\right|$ with equality only if there is equality both on the left in (30) and on the right in (31). But in this case we must have $\xi_{0}(\alpha)=\frac{\tau+1}{3}<\xi_{0}(\beta)$ and $\eta_{0}(\beta)=\frac{5 \tau-16}{3}>\eta_{0}(\alpha)$, when the two terms on the right of $(29)$ have the same sign. Thus in any case $f_{0}(\alpha)-f_{0}(\beta)$ has the sign of $\xi_{0}(\alpha)\left\{\eta_{0}(\alpha)-\eta_{0}(\beta)\right\}$ which has the sign of $(-)^{n_{1}-1}\left(\alpha_{-n_{1}}-\beta_{-n_{1}}\right)$. This proves (i). 
Again, if $n_{1} \geq n_{2}+3$, we have from (30) and (31)

$$
\left|\xi_{0}(\alpha)\left\{\eta_{0}(\alpha)-\eta_{0}(\beta)\right\}\right|<\left|\eta_{0}(\beta)\left\{\xi_{0}(\alpha)-\xi_{0}(\beta)\right\}\right|,
$$

so that by (29) $f_{0}(\alpha)-f_{0}(\beta)$ has the sign of $\eta_{0}(\beta)\left\{\xi_{0}(\alpha)-\xi_{0}(\beta)\right\}$ which has the sign of $\left(\alpha_{n_{2}}-\beta_{n_{2}}\right)$. This proves (ii).

(iii) is trivial, since each factor of $f_{0}(\alpha)$ is larger than the corresponding factor of $f_{0}(\beta)$. It is only useful when $n_{1}=n_{2}+2$, since otherwise it is contained in (i) or (ii).

We next consider the sequences satisfying the conditions of Corollary 1 . We use, for sequences $\left\{\alpha_{n}\right\}$, the notation of a square bracket and suffix already defined in $\S 1$.

Lemma 15. There are precisely an enumerable infinity of sequences $\left\{\alpha_{n}\right\}$ which do not contain any of the four subsequences 1000, 0111, 1011,0100. They are given by $\infty[0]_{\infty}, \quad \infty[0][10]_{\infty}, \quad \infty[1100]_{\infty}, \quad \infty[0][1100]_{\infty}, \quad \infty[0][1100]_{k}[10]_{\infty}, \quad \infty[0][1100]_{k} 11[01]_{\infty}$, where in the last two cases $k>0, k \geq 0$ respectively ${ }^{1}$, and the conjugate sequences.

Since the subsequence 1000 is excluded, any sequence containing 000 must extend to the left merely as $\infty_{[0]}[0]$ By conjugacy, similar results hold also to the left of 111 and the right of 101,010 .

Now suppose the sequence $\left\{\alpha_{n}\right\}$ contains 1100 . Then we select any one such occurrence, and take the longest subsequence of alternate pairs 00 and 11 containing it. This must take one of the forms

$$
00[1100]_{k} 11, \quad 00[1100]_{k}, \quad[1100]_{k} 11, \quad[1100]_{k},
$$

where $k$ is so interpreted as to include the possibility of extending to infinity on either or both sides. Suppose first that this subsequence is bounded on the left and begins with 00 . If the previous term is 1 , the one before it can neither be 1 (giving another pair, against our assumptions), nor 0 (giving the excluded subsequence 0100). Thus the previous term is 0 and, by the remarks above, the whole of the subsequence to the left must be simply $\infty_{\infty}[0]$. By conjugacy, we can now deal with the cases when the subsequence begins with 11 , or when it ends with 11 or 00 . Thus all

\footnotetext{
1 We suppose $k$ finite. If $k=\infty$ we may interpret the infinite repetition as extending in either direction, and obtain in each case a sequence which we are already considering explicitly. It should be noted, however, that we are not entitled to deduce the value of $M(f, P)$ simply by letting $k$ tend to $\infty$, since this is equivalent to inverting the order in a double limit.
} 
sequences containing 1100 (or, by conjugacy, 0011, 1001, 0110) are included in the list in the Lemma.

We now consider sequences satisfying the conditions of the Lemma which cio not include any of these four subsequences. We may suppose that such a sequence contains 00 , for it must contain at least one of the four conjugates $00,11,10,01$. Since the sequence cannot contain either 1000 or 1001 , the previous term must be 0 , and so the subsequence extends to the left as $\infty[0]$. Thus, either the sequence is $\infty[0]_{\infty}$ or it begins $\infty_{\infty}[0] 1$. In the latter case, since we can never have a further 00 or 11 by the previous argument, the sequence must be $\infty_{\infty}[0][10]_{\infty}$.

Conversely, all the sequences given in the lemma obey the conditions.

We have now to evaluate $M(f, P)$ for these various sequences. In each case the relevant point may be found from Lemma 11. In particular, the points corresponding to the first three sequences of Lemma 15 are respectively, in $(\xi, \eta)$-coordinates,

$$
\left(\frac{1+\tau}{3}, \frac{4-\tau}{3}\right),\left(\frac{1+2 \tau}{3}, \frac{4-\tau}{3}\right),\left(\frac{9}{13}+6, \frac{33-9 \tau}{13}\right)
$$

and so, in $(x, y)$-coordinates,

$$
\left(\frac{1}{3}, \frac{1}{3}\right),\left(\frac{\sqrt{13}+1}{3 \sqrt{13}}, \frac{\sqrt{13}+1}{2 \sqrt{13}}\right),\left(\frac{6}{13}, \frac{9}{13}\right) .
$$

Thus (by Theorem 7 of our previous paper) they represent the two types of point series at which $M(f, P)=M_{1}(f)=\frac{1}{3}$, and the point series at which $M(f, P)=M_{2}(f)=\frac{4}{13}$.

(We could also, of course, have obtained the minima for these point series in the same way as we shall do below for the other sequences of Lemma 15.)

We next consider the sequence $\infty[0][1100]_{\infty}$, defining, for reference, $\alpha_{0}$ as the last 0 in $\infty_{\infty}[0]$. We may clearly ignore any values of $\left|f_{n}\right|$ in which one factor is at least $\frac{1+\tau}{3}$ and the other at least $\frac{4-\tau}{3}$, since the product of these is $M_{1}(f)=\frac{1}{3}$. In particular, for this sequence we need only consider $\left|f_{n}(\alpha)\right|$ when $n>0$. Now by Lemmas 11 and 12

$$
\begin{aligned}
& f_{4 N+1}(\alpha)=-\left\{\frac{2 \tau-1}{3}-\frac{1+\tau^{-1}}{1-\tau^{-4}}\right\}\left\{\frac{2 \tau-5}{3}+\left(\tau^{-3}-\tau^{-4}\right) \sum_{0}^{N-1} \tau^{-4 n}\right\}, \\
& f_{4 N+2}(\alpha)=\left\{\frac{2 \tau+2}{3}-\frac{1+\tau^{-3}}{1-\tau^{-4}}\right\}\left\{\frac{8-2 \tau}{3}-\left(\tau^{-1}-\tau^{-4}\right) \sum_{0}^{N-1} \tau^{-4 n}-\tau^{-4 N-1}\right\},
\end{aligned}
$$




$$
\begin{aligned}
& f_{4 N+3}(\alpha)=-\left\{\frac{\tau-2}{3}+\frac{\tau^{-2}+\tau^{-3}}{1-\tau^{-4}}\right\}\left\{\frac{\tau-1}{3}-\left(\tau^{-1}-\tau^{-2}\right) \sum_{0}^{N} \tau^{-4 n}\right\}, \\
& f_{4 N+4}(\alpha)=\left\{\frac{1+\tau}{3}+\frac{\tau^{-1}+\tau^{-2}}{1-\tau^{-4}}\right\}\left\{\frac{4-\tau}{3}-\left(\tau^{-2}-\tau^{-3}\right) \sum_{1}^{N} \tau^{-4 n}\right\},
\end{aligned}
$$

where $N \geq 0$ in each case. It follows from these that $\operatorname{Min}\left|f_{4 N+2}(\alpha)\right|, \operatorname{Min}\left|f_{4 N+3}(\alpha)\right|$ and $\operatorname{Min}\left|f_{4 N+4}(\alpha)\right|$ are all taken at $N=\infty$, and are thus at least $\frac{4}{13}$ by comparison with $[1100]_{\infty}$. Again, $\operatorname{Min}\left|f_{4+1}(\alpha)\right|$ is taken at $N=0$ and is

$$
\left|f_{1}(\alpha)\right|=\frac{4 \tau-6}{13} \times \frac{2 \tau-5}{3}=\frac{38-8 \tau}{39}=M^{\prime}<\frac{4}{13}
$$

Thus for this sequence (and its four conjugates) $M(f, P)=M^{\prime}$.

We next consider the sequence $\infty_{\infty}[0][1100]_{k}[10]_{\infty}$, defining $\alpha_{0}$ as above. By conjugacy $\left|f_{n}(\alpha)\right|=\left|f_{4 k+1-n}(\alpha)\right|$; and as above we need not consider $\left|f_{n}(\alpha)\right|$ for $n \leq 0$. Thus we need only consider $f_{4 N+1}(\alpha), f_{4 N+2}(\alpha)(0 \leq N<k)$. For these we have, by Lemma 11,

$$
\begin{aligned}
& f_{4 N+1}(\alpha)=-\left\{\frac{2 \tau-1}{3}-\left(1+\tau^{-1}\right) \sum_{0}^{k-N-1} \tau^{-4 n}-\frac{\tau^{-4(k-N)}}{1-\tau^{-2}}\right\} \times\left\{\frac{2 \tau-5}{3}+\left(\tau^{-3}-\tau^{-4}\right) \sum_{0}^{N-1} \tau^{-4 n}\right\}, \\
& f_{4 N+2}(\alpha)=\left\{\frac{2 \tau+2}{3}-\left(1+\tau^{-3}\right) \sum_{0}^{k-N-1} \tau^{-4 n}-\frac{\tau^{-4(k-N)-1}}{1-\tau^{-2}}\right\} \times \\
& \times\left\{\frac{8-2 \tau}{3}-\left(\tau^{-1}-\tau^{-4}\right) \sum_{0}^{N-1} \tau^{-4 n}-\tau^{-4 n-1}\right\} .
\end{aligned}
$$

Now it is easy to see that $\left|f_{4 N+2}(\alpha)\right|$ is greater than the corresponiding $\left|f_{4+2}(\alpha)\right|$ for the sequence ${ }_{\infty}[0][1100]_{\infty}$ above, and so a fortiori greater than $\frac{4}{13}$; thus we may neglect it. Also, in $\left|f_{4 N+1}(\alpha)\right|$ both factors are increased by increasing $N$, so that

$$
\begin{array}{r}
M(f, P)=-f_{1}(\alpha)=\left\{\frac{2 \tau-5}{3}\right\}\left\{\frac{2 \tau-1}{3}-\left(1+\tau^{-1}\right)\left(\frac{1-\tau^{-4 k}}{1-\tau^{-4}}\right)-\frac{\tau^{-k k}}{1-\tau^{-2}}\right\} \\
=M^{\prime}+\tau^{-4 k}\left(\frac{11 \tau-23}{117}\right) .
\end{array}
$$

We argue similarly with $\infty[0][1100]_{k} 11[01]_{\infty}$. In this case conjugacy gives $\left|f_{n}(\alpha)\right|=\left|f_{4 k+3-n}(\alpha)\right|$, and we need consider only $f_{4 N+1}(\alpha)(0 \leq N \leq k)$ and $f_{4 N+4}(\alpha)$ $(0 \leq N<k)$. In these cases we find 


$$
\begin{aligned}
& f_{4 N+1}(\alpha)=-\left\{\frac{2 \tau-1}{3}-\left(1+\tau^{-1}\right) \sum_{0}^{k-N} \tau^{-4 n}-\frac{\tau^{-4(k-N)-3}}{1-\tau^{-2}}\right\}\left\{\frac{2 \tau-5}{3}+\left(\tau^{-3}-\tau^{-4}\right) \sum_{0}^{N-1} \tau^{-4 n}\right\} \\
& f_{4 N+4}(\alpha)=\left\{\frac{\tau+1}{3}+\left(\tau^{-1}+\tau^{-2}\right) \sum_{0}^{k-N} \tau^{-4 n}+\frac{\tau^{-4(k-N+1)}}{1-\tau^{-2}}\right\}\left\{\frac{4-\tau}{3}-\left(\tau^{-2}-\tau^{-3}\right) \sum_{0}^{N} \tau^{-4 n}\right\}
\end{aligned}
$$

Now $\left|f_{4 N+4}(\alpha)\right|$ is greater than the corresponding ||$_{4+44}(\alpha) \mid$ for the sequence ${ }_{\infty}[0][1100]_{\infty}$, and so a fortiori greater than $\frac{4}{13}$; thus we may neglect it. Again, in $\left|f_{4 N+1}(\alpha)\right|$ the first factor is

$$
\frac{2 \tau-1}{3}-\frac{1+\tau^{-1}}{1-\tau^{-4}}-\frac{\tau^{-4(k-N+1)}}{1-\tau^{-2}}\left(\tau-\frac{1+\tau^{-1}}{1+\tau^{-2}}\right)=\frac{4 \tau-6}{13}-\tau^{-4(k-N)-2}\left(\frac{\tau+5}{39}\right)
$$

and the second factor is $\frac{4 \tau-6}{13}-\tau^{-4 N}\left(\frac{47-14 \tau}{39}\right)$. It is clear from these two results that $\operatorname{Min}\left|f_{4+1}(\alpha)\right|$ is attained either at $N=0$ or $N=k$, the two extreme values of $N$. But

$$
\begin{gathered}
\left|f_{1}(\alpha)\right|=\frac{2 \tau-5}{3}\left\{\frac{4 \tau-6}{13}-\tau^{-4 k-2}\left(\frac{\tau+5}{39}\right)\right\}=M^{\prime}-\tau^{-4 k-2}\left(\frac{11 \tau-23}{117}\right), \\
\left|f_{4 k+1}(\alpha)\right|=\frac{2 \tau-5}{3}\left\{\frac{4 \tau-6}{13}-\tau^{-4 k}\left(\frac{47-14 \tau}{39}\right)\right\}=M^{\prime}-\tau^{-4 k-2}\left(\frac{11 \tau-23}{117}\right),
\end{gathered}
$$

so that

$$
M(t, P)=M^{\prime}-\tau^{-4 k-2}\left(\frac{11 \tau-23}{117}\right)
$$

If we write

$$
M_{k}^{\prime}=M^{\prime}+\left(-\tau^{-2}\right)^{k}\left(\frac{11 \tau-23}{117}\right)
$$

then (32) and (33) show that $M(f, P)=M_{2 k}^{\prime}$ or $M_{2 k+1}^{\prime}$ respectively; and $2 k, 2 k+1$ are the number of alternate pairs 11,00 not included in the semi-infinite ends $\infty_{\infty}[0]$ and $[01]_{\infty}$ or $[10]_{\infty}$.

In view of Corollaries 1 and 2, we have now proved that part of Theorem 2 which is concerned with the top line of (26). This is the most interesting part of the Theorem, since it shows that $f$ does not behave in the same simple way as the other quadratic forms that have been investigated. It will be clear to the reader that if we had only been concerned to prove this much, we could have simplified the work in several places (as well as omitting Lemma 14, which is only useful later). We shall in fact sketch an alternative proof of Lemma 15 - the crux of 
the argument - on lines which make the cause of it much clearer and enable us to dispence with the $\alpha_{n}$ altogether.

We define the region $\mathfrak{R}_{j k}^{i}(i, j, k=1,2,3,4)$ as being the set of points $P$ for which $P \in \mathcal{R}_{i}, \quad T P \in \mathcal{R}_{j}(\bmod 1), T^{-1} P \in \boldsymbol{R}_{k}(\bmod 1)$. It may be verified that there are just 16 such regions, and (for admissible points) any one of them is defined by specifying $\alpha_{-2}, \alpha_{-1}, \alpha_{0}$ and $\alpha_{1}$. Moreover, if $P$ is admissible and $P^{(0)} \in \boldsymbol{R}_{j k}^{i}$, then $P^{(1)} \in \boldsymbol{R}_{h i}^{j}$, where $h=1$ or 3 if $j=1$ or $2, h=2$ or 4 if $j=3$ or 4 . Corollary 1 now becomes

Let $P$ be admissible. Then the necessary and sufficient condition for $M(f, P)>$ $>\frac{41 \tau-133}{9}$ is that no $P^{(n)}$ should lie in any of $\mathfrak{R}_{12}^{1}, \mathfrak{R}_{13}^{2}, \boldsymbol{R}_{42}^{3}$ or $\boldsymbol{R}_{43}^{4}$.

In this form, the result can easily be proved in the same way as Lemma 4 . It remains to show that there are only an enumerable infinity of point-series satisfying this condition. We group the remaining twelve regions as follows

$$
\begin{aligned}
& S_{1}: \quad R_{33}^{2}, \quad R_{22}^{3}, \\
& S_{2}: R_{21}^{3}, \quad R_{34}^{2} \text {, } \\
& S_{3}: \quad R_{32}^{1}, \quad R_{14}^{2}, \quad R_{23}^{4}, \quad R_{41}^{3} \text {, } \\
& S_{4}: R_{31}^{1}, R_{24}^{4}, \\
& S_{5}: R_{11}^{1}, \quad R_{44}^{4} \text {, }
\end{aligned}
$$

It may now be easily verified that

(i) if $P^{(0)}$ lies in a region of $S_{i}$, then $P^{(1)}$ lies in a region of some $S_{j}$ with $j \leq i$

(ii) If $P^{(0)}, P^{(1)}$ lie in regions of the same $S_{i}$, then the region in which $P^{(0)}$ lies uniquely determines the region in which $P^{(1)}$ lies.

From these it is clear that if $n_{i}$ (which can be $\pm \infty$ ) is the greatest $n$ for which $P^{(n)}$ lies in some $S_{j}$ with $j \geq i$, then the whole route, and so $P^{(0)}$, is determined by the integers $n_{i}(i=2,3,4,5)$ and the regions in which $P^{\left(n_{i}\right)}, P^{\left(n_{i}+1\right)}$ lie. Thus the routes depend on the $n_{i}$ and a finite number of choices, and so form an enumerable set.

A more detailed consideration of the possibilities (which are most simply represented diagramatically) gives the whole of Lemma 15. It will be seen that though, for example, $\boldsymbol{R}_{11}^{1}$ and $\boldsymbol{R}_{33}^{2}$ are conjugate, they play essentially different roles - which is bound up with the fact that $\left(\begin{array}{c}0^{-}-1 \\ 1\end{array}\right)$ does not commute with $T$. 
We have now to consider sequences which do not satisfy the conditions of Corollary 1.

Lemma 16. For the point series defined by the sequence $\infty_{\infty}[0011] 0110[0011]_{\infty}$ we have $M(f, P)=M^{\mathrm{IV}}$. Moreover, the last sentence of Theorem 2 holds.

We shall for convenience define $\alpha_{0}$ as the first 0 in [0011 $]_{\infty}$. Now, by Corollary 2, we need only consider in Lemma 12 the cases $n=-3,0$; and these are conjugate points which give the same $\left|f_{n}(\alpha)\right|$, so that we need only consider the latter. Thus

$$
\begin{aligned}
M(f, P) & =\left\{\frac{\tau+1}{3}+\frac{\tau^{-2}+\tau^{-3}}{1-\tau^{-4}}\right\}\left\{\frac{4-\tau}{3}-\tau^{-2}+\tau^{-3}+\frac{\tau^{-5}-\tau^{-6}}{1-\tau^{-4}}\right\} \\
& =\frac{4 \tau+7}{13} \times \frac{113 \tau-371}{13}=\frac{51 \tau-165}{13}=M^{\mathrm{IV}} .
\end{aligned}
$$

The other result in the Lemma follows at once by the same argument as the corresponding result in Theorem 1, since both the semi-infinite ends of the sequence giving $\boldsymbol{M}^{\mathrm{IV}}$ are made up of an infinite repetition of the same subsequence (unlike the sequences of Lemma 15).

We now define a sequence as strongly admissible if for the corresponding point series we have $M(f, P) \geq M^{\mathrm{IV}}$ and the sequence contains one of the subsequences $1000,0111,1011,0100$. We now obtain some properties of strongly admissible sequences by using Lemma 14, noting that $M^{\mathrm{IV}}>\frac{1}{4}$.

Lemma 17. Suppose a strongly admissible sequence contains 1000. Then it contains it as part of the subsequence 0011011000110; and similarly for the conjugate subsequences.

For convenience of reference we take $\alpha_{0}$ to be the second 0 of 1000. An immediate application of Lemma 14 (i) shows that $\alpha_{-3}=1$. We next see that $\alpha_{-4}=0$ and $\alpha_{2}=1$, since any other pair of assumptions conflicts with one of the parts of Lemma 14. Repeating this argument, we have further $\alpha_{-5}=1, \alpha_{3}=1$. Thus 1000 is certainly contained in 101100011. By conjugacy, 0111, 1011 and 0100 must be contained respectively in 010011100,011011000 and 100100111.

Now the sequence we are working with cannot have $\alpha_{4}=1$, since it would then contain 0111 in a manner inconsistent with our last result; therefore we must have $\alpha_{4}=0$. Since the sequence now contains 1011, we can insert the result for it, and this gives us the whole of Lemma 17 . 
Now let us consider any strongly admissible sequence $\left\{\alpha_{n}\right\}$ containing 1000 . Let $-n_{1}, n_{2}$ be the greatest negative and least positive values of $n$ for which $\alpha_{n}$ is not the same as for the basic sequence in Lemma 16. Thus by Lemma 17, $n_{1} \geq 9, n_{2} \geq 5$. We see at once that the cases $n_{1} \equiv 0,2(\bmod 4)$ or $n_{2} \equiv 0,2(\bmod 4)$ are impossible, since one of the subsequences 1011, 0100, 1000, 0111 would then occur in a manner inconsistent with Lemma 17.

Applying Lemma 14, we find also

(i) if $n_{1}=1(\bmod 4)$, then $n_{1}>n_{2}+1$;

(ii) if $n_{2} \equiv 3(\bmod 4)$, then $n_{1}<n_{2}+3$;

(iii) we cannot have $n_{1}=1(\bmod 4), n_{2} \equiv 3(\bmod 4)$;

and to these we may add the conjugate results

$$
\begin{aligned}
& \text { (iv) if } n_{2} \equiv 1(\bmod 4) \text {, then } n_{1}<n_{2}+7 \\
& \text { (v) if } n_{1} \equiv 3(\bmod 4) \text {, then } n_{1}>n_{2}+5 ; \\
& \text { (vi) we cannot have } n_{2}=1(\bmod 4), n_{1}=3(\bmod 4) \text {. }
\end{aligned}
$$

Thus the only possibility is

$$
n_{\mathbf{1}} \equiv n_{2} \equiv 1(\bmod 4), n_{1}=n_{2}+4 .
$$

Let us write, for convenience, $n_{2}=4 k+1, n_{1}=4 k+5$ where $k>0$. Then the portion of $\left\{\alpha_{n}\right\}$ which is the same as the basic sequence is $[0011]_{k} 01100[0110]_{k}$. We consider how this may be continued on the right.

We know that $\alpha_{4 k+1}=\alpha_{n_{2}}=1$, by definition. Now there are two possibilities for $\alpha_{\mathbf{4}+2+2}$ :

(i) $\alpha_{4 k+2}=1$. We have now the subsequence 1011; and by Lemma 17 and (35) the sequence must continue (starting at $\alpha_{4 k+1}$ ) $1100[0110]_{k}$, with the same value of $k$ as before.

(ii) $\alpha_{4 k+2}=0$. It may easily be seen that the rest of the sequence is simply $[10]_{\infty}$, since any other continuation would contain a subsequence 0100 or 1011 in a manner inconsistent with Lemma 17.

Thus, using eonjugacy, we have

Lemma 18. The strongly admissible sequences are precisely

$$
\begin{gathered}
\infty[0011] 0110[0011]_{\infty}, \quad \infty\left[0110[0011]_{k}\right]_{\infty}, \quad \infty[0]\left[[0011]_{k} 0110\right]_{\infty}, \\
\infty[0]\left[[0011]_{k} 0110\right]_{h}[0011]_{k}[10]_{\infty}
\end{gathered}
$$

(where $h>0, k>0)^{1}$, and their conjugates.

1 We suppose $h, k$ finite, for the reasons already stated in the footnote to Lemma 15. 
The first of these sequences has been discussed in Lemma 16. It therefore only remains to find the values of $M(f, P)$ for the other three and to arrange these values in order. We shall write the minima for the last three sequences as $\boldsymbol{M}_{k}^{\prime \prime}, \boldsymbol{M}_{k}^{\prime \prime \prime}, \boldsymbol{M}_{k, h}^{\prime \prime \prime}$ respectively.

We first consider $M_{k}^{\prime \prime}$. By conjugacy, all the values of $\left|f_{n}(\alpha)\right|$ which we have to consider (after Corollary 2) are equal, and we have

$$
\begin{aligned}
M_{k}^{\prime \prime} & =\left\{\frac{1+\tau}{3}+\frac{\tau^{-2}+\tau^{-3}}{1-\tau^{-4}}+\frac{\tau^{-4 k-1}-\tau^{-4 k-3}}{1-\tau^{-4 k-4}}\right\}\left\{\frac{4-\tau}{3}+\frac{\tau^{-1}-\tau^{-2}}{1-\tau^{-4}}-\frac{\tau^{-1}-\tau^{-3}}{1-\tau^{-4 k-4}}\right\} \\
& =\left\{\frac{7+4 \tau}{13}+\frac{(9 \tau+3) \tau^{-4 k-4}}{1-\tau^{-4 k-4}}\right\}\left\{\frac{19-4 \tau}{13}-\frac{30-9 \tau}{1-\tau^{-4 k-4}}\right\} \\
& =\frac{9}{13}+\frac{51 \tau+21}{13} \times \frac{\tau^{-4 k-4}}{1-\tau^{-4 k-4}}-\frac{174-51 \tau}{13} \times \frac{1}{1-\tau^{-4 k-4}}-\frac{9 \tau^{-4 k-4}}{\left(1-\tau^{-4 k-4}\right)^{2}} \\
& =-\frac{177}{26}+\frac{51 \sqrt{13}}{26}\left(\frac{1+\tau^{-4 k-4}}{1-\tau^{-4 k-4}}\right)-\frac{9 \tau^{-4 k-4}}{\left(1-\tau^{-4 k-4}\right)^{2}} .
\end{aligned}
$$

$M_{k}^{\prime \prime}$ is obviously rational, since it is generated by a periodic sequence, i.e. a rational point. Also, by Lemma 14, or directly from (36),

$$
M_{k}^{\prime \prime}>M_{k+1}^{\prime \prime}, \quad M_{k}^{\prime \prime}>M^{\mathrm{IV}}, \quad \lim M_{k}^{\prime \prime}=M^{\mathrm{IV}}
$$

We next consider $M_{k}^{\prime \prime \prime}$, taking for convenience $\alpha_{0}$ to be the last 0 in $\infty[0]$. By Corollary 2 we need only consider those $f_{n}(\alpha)$ for which

$$
n \equiv 4 k+2(\bmod 4 k+4), n>0 \text { or } n \equiv 1(\bmod 4 k+4), n>4 k+4 ;
$$

and it now follows from Lemma 14 that the least $\left|f_{n}(\alpha)\right|$ is given by $n=4 k+2$; and consequently that

We have also

$$
M_{k}^{\prime \prime}>M_{k}^{\prime \prime \prime}>M_{k+1}^{\prime \prime}, \quad \lim M_{k}^{\prime \prime \prime}=M^{\mathrm{IV}}
$$

$$
\begin{aligned}
M_{k}^{\prime \prime \prime} & =-\left\{\frac{\tau+1}{3}+\frac{\tau^{-1}+\tau^{-2}}{1-\tau^{-4}}+\frac{1-\tau^{-2}}{1-\tau^{-4 k-4}}-\tau\right\}\left\{\frac{4-\tau}{3}+\tau^{-1}-\frac{\left(\tau^{-2}-\tau^{-3}\right)\left(1-\tau^{-4 k}\right)}{1-\tau^{-4}}\right\} \\
& =\left\{\frac{113-32 \tau}{13}-\frac{3(\tau-3) \tau^{-4 k-4}}{1-\tau^{-4 k-4}}\right\}\left\{\frac{7 \tau-17}{13}+\frac{(5 \tau-14) \tau^{-4 k}}{39}\right\} \\
& =M^{\mathrm{IV}}-\frac{174-51 \tau}{13} \times \frac{\tau^{-4 k-4}}{1-\tau^{-4 k-4}}+\frac{(7-\tau) \tau^{-4 k}}{39}-\frac{47-14 \tau}{13} \times \frac{\tau^{-4 k}}{1-\tau^{-4 k-4}} \\
& =M^{\mathrm{IV}}+\tau^{-4 k}(7-\tau)\left(\frac{1}{39}-\frac{2 \tau^{-4}}{1-\tau^{-4 k-4}}\right) .
\end{aligned}
$$


Finally we must consider $\boldsymbol{M}_{k, h}^{\prime \prime \prime}$. If we define $\alpha_{0}$, as before, as the last 0 in ${ }_{\infty}[0]$, arguments similar to those above show that $M_{k, h}^{\prime \prime \prime}=\left|f_{4 k+2}(\alpha)\right|$, and we may deduce by Lemma 14 that

$$
M_{k}^{\prime \prime \prime}>M_{k, h}^{\prime \prime \prime}>M_{k+1}^{\prime \prime}, \quad M_{k, h+1}^{\prime \prime \prime}>M_{k, h}^{\prime \prime \prime}, \quad \lim _{h \rightarrow \infty} M_{k, h}^{\prime \prime \prime}=M_{k}^{\prime \prime \prime} .
$$

The inequalities (37), (38) and (40), with the results already obtained, complete the proof of Theorem 2. It only remains to calculate $M_{k, h}^{\prime \prime \prime}$, and we find

$$
\begin{aligned}
M_{k, h}^{\prime \prime \prime} & =\left\{\tau-\frac{\tau+1}{3}-\left(1-\tau^{-4 h(k+1)}\right)\left(\frac{\tau^{-1}+\tau^{-2}}{1-\tau^{-4}}+\frac{1-\tau^{-2}}{1-\tau^{-4 k-4}}\right)-\frac{\tau^{1-4 h(k+1)}}{1-\tau^{-2}}\right\} \times \\
\times\left\{\frac{4-\tau}{3}-\frac{\left(\tau^{-2}-\tau^{-3}\right)\left(1-\tau^{-4 k}\right)}{1-\tau^{-4}}+\tau^{-1}\right\} & \\
& =M_{k}^{\prime \prime \prime}\left(1-\tau^{-4 h(k+1)}\right)-\tau^{-4 h(k+1)}\left(\frac{6 \tau-9}{13}+\frac{(11 \tau-23) \tau^{-4 k}}{117}\right)
\end{aligned}
$$

3. If we apply these methods to a general form, we can no longer obtain as much. In what follows, we assume that $f(x, y)=a x^{2}+b x y+c y^{2}$ is an indefinite binary quadratic form with integral coefficients, which does not represent zero.

It is natural to conjecture that $M_{1}(f)$ is rational and assumed at rational points though not necessarily there alone; and it seems probable that this can be proved by the methods of this and our previous paper. All we have been able to prove, however, are the following simple results.

Theorem L. (i) To any point $P$ there corresponds a point $P_{1}$ such that $M(f, P)=$ $=M\left(f, P_{1}\right)$ and $M\left(f, P_{1}\right)$ is attained .

(ii) The set of values of $M(f, P)$, as $P$ varies, is closed.

The second part of this generalizes Heinhold's result (our 'Theorem $A$ ) that $M_{1}(f)$ is an assumed upper bound.

Let $T$ be the fundamental automorph of $f \cdot$ For any point $P$ and any integer $n$ we define $P^{(n)}=\left(x^{(n)}, y^{(n)}\right)$ as the point congruent to $T^{n} P$ in the unit square $0 \leq x, y<1$. Now from Theorem B,

$$
M(f, P)=\operatorname{Min}_{n} \operatorname{Min}_{X, Y}\left|f\left(X+x^{(n)}, Y+y^{(n)}\right)\right|
$$

taken over all $n$ and a certain finite set of integer pairs $(X, Y)$ depending only on $f$ and $T$. We may choose a subsequence $\left\{n_{i}\right\}$ of the $n$ such that

$$
M(f ; P)=\lim _{i \rightarrow \infty} \operatorname{Min}_{x, y}\left|f\left(X+x^{\left(n_{i}\right)}, Y+y^{\left(n_{i}\right)}\right)\right|
$$


We may without loss of generality suppose that all the $n_{i}$ are distinct; for this is clearly possible if $M(f, P)$ is unattained, while if $M(f, P)$ is attained the theorem is trivial. Now let $P_{1}=\left(x_{1}, y_{1}\right)$ be any point of condensation of the $P^{\left(n_{i}\right)}$. Then it follows from (43) that

$$
M(f, P)=\operatorname{Min}_{X, Y}\left|f\left(X+x_{1}, Y+y_{1}\right)\right| \geq M\left(f, P_{1}\right) .
$$

On the other hand, since cvery $P_{1}^{(n)}$ is a point of condensation of the $P^{(n)}$, it follows easily from (42), applied to $P_{1}$, that $M(f, P) \leq M\left(f, P_{1}\right)$. These results together prove (i).

The proof of (ii) is similar. We suppose a sequence of points $P_{i}$ such that

$$
\lim _{i \rightarrow \infty} M\left(f, P_{i}\right)=k,
$$

and we have to find a point $P$ for which $M(f, P)=k$. After (i), we may assume without loss of generality that every $M\left(f, P_{i}\right)$ is attained. Thus for each $i$ wo can find an $n_{i}$ for which

$$
M\left(f, P_{i}\right)=\operatorname{Min}_{X, Y}\left|f\left(X+x_{i}^{\left(n_{i}\right)}, Y+y_{i}^{\left(n_{i}\right)}\right)\right|,
$$

in virtue of (i). Now let $P$ be any point of condensation of the $P_{i}^{\left(n_{i}\right)}$. By an argument exactly analogous to that above, we see that $M(f, P)=k$, and this proves the theorem.

Theorem M. Given any $\varepsilon>0$, there is a rational point $P$ such that $M(f, P)>$ $>M_{1}(f)-\varepsilon$.

Suppose that the linear factorization of $f$ is $f=\xi \eta$, and suppose that $\tau>1$ is the fundamental unit of $k(\sqrt{d})$, so that $T$ changes $\xi, \eta$ into $\tau \xi, \tau^{\prime} \eta$, where $\tau \tau^{\prime}= \pm 1$. Since $M_{1}(f)$ is attained, there is a $P_{0}$ such that $M\left(f, P_{0}\right)=M_{1}(f)$. We shall denote the coordinates of any $P_{i}$ or $P_{i}^{(n)}$ by the corresponding suffixes and indices; and we give $(X, Y)$ the same meaning as in the proof of Theorem $\mathrm{I}$. We choose $\varepsilon_{1}>0$ so that, for any pair of points $P_{2}, P_{3}$ with $\left|x_{2}\right| \leq \operatorname{Max}|X|+1,\left|y_{2}\right| \leq \operatorname{Max}|Y|+1$ and $\left|\xi_{2}-\xi_{3}\right|<\varepsilon_{1},\left|\eta_{2}-\eta_{3}\right|<\varepsilon_{1}$, wo have $\left|\xi_{2} \eta_{2}-\xi_{3} \eta_{3}\right|<\varepsilon$.

Now consider the $P_{0}^{(n)}$. We can choose two values $n_{1}, n_{2}$ of $n$, with $n_{2}>n_{1}$, such that $\left|\xi_{0}^{\left(n_{1}\right)}-\xi_{0}^{\left(n_{2}\right)}\right|<\frac{1}{2} \varepsilon_{1},\left|\eta_{0}^{\left(n_{1}\right)}-\eta_{0}^{\left(n_{2}\right)}\right|<\frac{1}{2} \varepsilon_{1}$. Lot $A$ be the integral point such that

$$
T^{n_{2}-n_{1}} P_{0}^{\left(n_{1}\right)}=P_{0}^{\left(n_{2}\right)}+A \text {. }
$$

We define the point $P_{1}$ to satisfy

$$
T^{n_{2}-n_{1}} P_{1}=P_{1}+A
$$


so that $P_{1}$ is rational and mimics $P_{0}^{\left(n_{1}\right)}$ under small positive powers of $T$. Then

$$
\xi_{1}=\xi_{0}^{\left(n_{1}\right)}+\frac{\xi_{0}^{\left(n_{1}\right)}-\xi_{0}^{\left(n_{2}\right)}}{\tau^{n_{2} n_{1}}-1}, \eta_{1}=\eta_{0}^{\left(n_{1}\right)}+\frac{\eta_{0}^{\left(n_{1}\right)}-\eta_{0}^{\left(n_{2}\right)}}{\tau^{\prime n_{2}-n_{1}}-1} .
$$

We can clearly assume that $\tau^{n_{2}-n_{1}}>2$, so that these give

$$
\left|\xi_{1}-\xi_{0}^{\left(n_{1}\right)}\right|<\varepsilon_{1} \tau^{n_{1} \cdots n_{2}}, \quad\left|\eta_{1}-\eta_{0}^{\left(n_{1}\right)}\right|<\varepsilon_{1}
$$

Hence, for $n_{1} \leq n \leq n_{2}$, we have

$$
\left|\xi_{1}^{\left(n-n_{1}\right)}-\xi_{0}^{(n)}\right|<\varepsilon_{1}, \quad\left|\eta_{1}^{\left(n-n_{1}\right)}-\eta_{0}^{(n)}\right|<\varepsilon_{1} .
$$

By the same argument as in the proof of Theorem $\mathrm{L}$ we now deduce that $M\left(f, P_{1}\right)>$ $>M\left(f, P_{0}\right)-\varepsilon$, which proves the theorem.

It is clear from Theorem $\mathrm{L}$ that if $k$ is any positive real number not a value of $M(f, P)$ for some $P$, then we can carry through the arguments of the previous sections in a finite number of steps; and that all the points with $M(f, P)>k$ can be represented by sequences $\left\{\alpha_{n}\right\}$, subject only to local conditions similar to those in Lemma 5. Moreover, there will be an algebraic representation of the point in terms of the $\alpha_{n}$, similar to those of Lemmas 7 and 11. For any given form, it is now possible to lay down selection rules (cf. Lemmas 9 and 14), and so to obtain the complete enumerable sequence of minima of $M(f, P)$. However, since these selection rules may be of arbitrary complexity, we do not see how to prove any general result by these methods. Conversely, though wo can lay down selection rules corresponding to almost any behaviour of the $M(f, P)$, it is impossible to work back from them to a form. The rest of what we have to say in this section is therefore conjectural.

The normal result is that of Theorem 1; to this type also belong the forms $x^{2}+x y-y^{2}, x^{2}-2 y^{2}$, investigated by Davenport and Varnavides. This is the behaviour we should have expected a priori, by analogy with almost all the results that have been obtained in the Geometry of Numbers. We must consider what deviations from it are likely, and what peculiarities of the form would be needed to bring them about. We believe that the following two statements are true:

(i) $M_{1}(f)$ is rational, isolated and taken at rational points (though possibly also at irrational points in the field $k(\sqrt{d}))$;

(ii) $M_{2}(f)$ exists, and is taken at points in the field $k(\sqrt{d})$;

and that no stronger results are true in general. In particular, there may well exist forms for which $M_{2}(f)$ is not isolated. 
In support of this last statement, consider the effect of replacing $-\tau$ by $+\tau$ in the formulae of Lemmas 7 and 8. It is easily seen that then $M_{1}(f)$ would be given by the sequence $\infty_{\infty}[1]_{\infty}$ and $M_{2}(f)$ by the sequence $\infty_{\infty}[1] 0[1]_{\infty}$; and that the set of values of $M(f, P)$ in any interval $M_{2}(f)>M(f, P)>M_{2}(f)-\varepsilon$ would have the cardinal number of the continuum. The suggested alteration is not obviously implausible, but it is not clear how to find a form corresponding to it.

The reader will note that the proof of Theorem 2 carries over to the general form $x^{2}+(2 n-1) x y-y^{2}$, as Theorem 7 of our provious paper would suggest. Apart from these, the only form we know whose behaviour does not follow the pattern of Theorem 1 is $x^{2}+x y-15 y^{2}$. In Theorem 11 of our previous paper we proved that, for this form, $M_{2}(f)$ is taken at both rational and irrational points; but we have not constructed the complete enumerable sequence of minima, since it is already clear from the proof that $M_{2}(f)$ is isolated.

It would be of interest to find further examples of forms for which there are only an enumerably infinite number of values of $M(f, P)$ in a small neighbourhood of $M^{\prime}=\overline{\lim } M(f, P)$. For any such form, supposing reasonably simple selection rules, $M^{\prime}$ must arise from a sequence $\left\{\alpha_{n}\right\}$ whose semi-infinite ends are periodic. Moreover, the periods on the right and left must be different, since otherwise we can apply the argument by which we proved the last assertion of Theorem 1; thus, in particular, the reversed sequence cannot give the same minimum $M^{\prime}$.

An examination of the proof of Theorem 2, especially Lemma 11 and the second proof of Lemma 15, suggests two possible reasons for the irreversibility of $\left\{\alpha_{n}\right\}$ when $f=x^{2}+3 x y-y^{2}$ : (i) the fundamental unit $\tau$ has norm - 1 ; (ii) the form is ambiguous, and so possesses an automorph $U=\left(\begin{array}{rr}0 & -1 \\ 1 & 0\end{array}\right)$ of finite order which does not commute with the fundamental automorph $T$. The second reason is more likely to be the deciding factor, since it introduces a lack of symmetry into the definition of conjugate points without destroying the obvious symmetry in $T$ and $T^{-1}$. It seems, however, that if $M_{1}(f)$ is taken at any of the points $\left(\frac{1}{2}, \frac{1}{2}\right),\left(0, \frac{1}{2}\right)$ or $\left(\frac{1}{2}, 0\right)$, as with the forms investigated by Davenport and Varnavides, this introduces enough extra symmetry to prevent any anomalous behaviour.

We list below those forms with small discriminant which have either of the two properties (i) and (ii) given above. We omit forms which may be shown by the results of Heinhold [1] or Barnes [1] to take their first minimum at $\left(\frac{1}{2}, \frac{1}{2}\right),\left(0, \frac{1}{2}\right)$ or $\left(\frac{1}{2}, 0\right)$. Table 1 gives forms with $D=1(\bmod 4)$, and Table 2 forms with $D / 4 \equiv 2$ $(\bmod 4)$. In each table, the first section gives forms with both properties (i) and (ii); 
TABLE 1 .

$D=d=1(\bmod 4), t^{2}-D u^{2}= \pm 4$.

\begin{tabular}{|c|r|r|r|}
\hline$f$ & $d$ & $t$ & \multicolumn{1}{|c|}{$u$} \\
\hline $2 x^{2}+5 x y-2 y^{2}$ & 41 & 64 & 10 \\
$3 x^{2}+5 x y-3 y^{2}$ & 61 & 39 & 5 \\
$4 x^{2}+3 x y-4 y^{2}$ & 73 & 2136 & 250 \\
$4 x^{2}+5 x y-4 y^{2}$ & 89 & 1000 & 106 \\
$2 x^{2}+9 x y-2 y^{2}$ & 97 & 11208 & 1138 \\
$5 x^{2}+3 x y-5 y^{2}$ & 109 & 261 & 25 \\
$4 x^{2}+5 x y-4 y^{2}$ & 113 & 1552 & 146 \\
$2 x^{2}+11 x y-2 y^{2}$ & 137 & 3488 & 298 \\
$5 x^{2}+7 x y-5 y^{2}$ & 149 & 61 & 5 \\
$3 x^{2}+11 x y-3 y^{2}$ & 157 & 213 & 17 \\
$5 x^{2}+9 x y-5 y^{2}$ & 181 & 1305 & 97 \\
$4 x^{2}+11 x y-4 y^{2}$ & 185 & 136 & 10 \\
$2 x^{2}+13 x y-2 y^{2}$ & 185 & 136 & 10 \\
\hline $3 x^{2}+11 x y-3 y^{2}$ & 205 & 43 & 3 \\
$5 x^{2}+11 x y-5 y^{2}$ & 221 & 15 & 1 \\
$2 x^{2}+17 x y-2 y^{2}$ & 305 & 978 & 56 \\
\hline $3 x^{2}+13 x y-5 y^{2}$ & 229 & 15 & 1 \\
$4 x^{2}+15 x y-11 y^{2}$ & 4101 & 40 & 2 \\
$3 x^{2}+19 x y-7 y^{2}$ & 445 & 21 & 1 \\
\hline
\end{tabular}

the second section, forms with (ii) but not (i); the third section, forms with (i) but not (ii).

4. Many of the results of this and our previous paper may be extended to norm-forms in $n$ variables, both of real and of complex fields. For a complete set of units of the field defines an infinite Abelian group $\mathbf{G}$ of automorphs of the form; and the part of the fundamental domain $\mathcal{R}$ of $\mathbf{G}$ in which the form is bounded is itself bounded. This is the analogue of Theorem $B$; the exact algebraic formulation presents no difficulties.

Theorem D is now true as it stands, and Theorem C needs only a few obvious alterations. In particular, it should be noted that to fix $P$ we do not need to know the behaviour of $P$ under every transform of $\mathbf{G}$, but only under those of a cyclic subgroup of $\mathbf{G}$.

Most of our general theorems follow from Theorems B and C, with the HeineBorel Theorem; thus they carry over immediately to the. general norm-form. In 
TABLE 2.

$D=4 d, d \equiv 2(\bmod 4), t^{2}-D u^{2}= \pm 4$.

\begin{tabular}{|c|c|c|c|}
\hline$f$ & $d$ & $t$ & $u$ \\
\hline $7 x^{2}+6 x y-7 y^{2}$ & 58 & 198 & 13 \\
\hline $3 x^{2}+14 x y-3 y^{2}$ & 58 & 198 & 13 \\
\hline $5 x^{2}+14 x y-5 y^{2}$ & 74 & 86 & 5 \\
\hline $9 x^{2}+10 x y-9 y^{2}$ & 106 & 8010 & 389 \\
\hline $5 x^{2}+18 x y-5 y^{2}$ & 106 & 8010 & 389 \\
\hline $7 x^{2}+18 x y-7 y^{2}$ & 130 & 114 & 5 \\
\hline $11 x^{2}+6 x y^{--11 y^{2}}$ & 130 & 114 & 5 \\
\hline $3 x^{2}+22 x y-3 y^{2}$ & 130 & 114 & 5 \\
\hline $7 x^{2}+22 x y-7 y^{2}$ & 170 & 26 & 1 \\
\hline $9 x^{2}+22 x y-9 y^{2}$ & 202 & 6282 & 221 \\
\hline $11 x^{2}+18 x y-11 y^{2}$ & 202 & 6282 & 221 \\
\hline $13 x^{2}+14 x y-13 y^{2}$ & 218 & 502 & 17 \\
\hline $5 x^{2}+22 x y-5 y^{2}$ & 146 & 290 & 12 \\
\hline $3 x^{2}+26 x y-3 y^{2}$ & 178 & 3202 & 120 \\
\hline $5 x^{2}+26 x y-5 y^{2}$ & 194 & 390 & 14 \\
\hline $5 x^{2}+38 x y-5 y^{2}$ & 386 & 223110 & 5678 \\
\hline $11 x^{2}+34 x y-11 y^{2}$ & 410 & 162 & 4 \\
\hline $3 x^{2}+16 x y-6 y^{2}$ & 82 & 18 & 1 \\
\hline $3 x^{2}+28 x y-10 y^{2}$ & 226 & 30 & 1 \\
\hline $6 x^{2}+20 x y-21 y^{2}$ & 226 & 30 & 1 \\
\hline $9 x^{2}+16 x y-18 y^{2}$ & 226 & 30 & 1 \\
\hline $3 x^{2}+32 x y-6 y^{2}$ & 274 & 2814 & $8 \tilde{5}$ \\
\hline $3 x^{2}+34 x y-19 y^{2}$ & 346 & 186 & 5 \\
\hline $6 x^{2}+32 x y-15 y^{2}$ & 346 & 186 & 5 \\
\hline $3 x^{2}+40 x y-14 y^{2}$ & $44: 2$ & 42 & 1 \\
\hline $6 x^{2}+40 x y-7 y^{2}$ & 442 & 42 & 1 \\
\hline
\end{tabular}

particular, this is true of the isolation Theorem $G$; the lack of this has cost previous writers some trouble.

All that have to be abandoned are the arithmetical Theorems $\mathrm{H}, \mathrm{J}$ and $\mathrm{K}$. It is possible to obtain analogous results in more variables, but these are so tedious and require the consideration of so many special cases as to make them almost useless. However, these theorems, though convenient, are not essential to our method, and it is perfectly possible to work with the hyperboloids direct.

Trinity College, Cambridge. 